Article

\title{
The Newly Discovered Neoproterozoic Aillikite Occurrence in Vinoren (Southern Norway): Age, Geodynamic Position and Mineralogical Evidence of Diamond-Bearing Mantle Source
}

\author{
Dmitry R. Zozulya ${ }^{1, *}{ }^{\mathbb{C}}$, Kåre Kullerud ${ }^{2} \mathbb{(}$, Enrico Ribacki ${ }^{3}$, Uwe Altenberger ${ }^{3}$, \\ Masafumi Sudo ${ }^{3}$ and Yevgeny E. Savchenko ${ }^{1}$ (i) \\ 1 Kola Science Centre, Geological Institute, Russian Academy of Science, 184209 Apatity, Russia; \\ evsav@geoksc.apatity.ru \\ 2 Norwegian Mining Museum, 3616 Kongsberg, Norway; kk@bvm.no \\ 3 Institute of Geosciences, University of Potsdam, 14476 Potsdam-Golm, Germany; \\ ribacki@uni-potsdam.de (E.R.); altenber@uni-potsdam.de (U.A.); \\ Masafumi.Sudo@geo.uni-potsdam.de (M.S.) \\ * Correspondence: zozulya@geoksc.apatity.ru; Tel.: +7-81555-79742
}

Received: 21 October 2020; Accepted: 16 November 2020; Published: 18 November 2020

check for updates

\begin{abstract}
During the period 750-600 Ma ago, prior to the final break-up of the supercontinent Rodinia, the crust of both the North American Craton and Baltica was intruded by significant amounts of rift-related magmas originating from the mantle. In the Proterozoic crust of Southern Norway, the 580 Ma old Fen carbonatite-ultramafic complex is a representative of this type of rocks. In this paper, we report the occurrence of an ultramafic lamprophyre dyke which possibly is linked to the Fen complex, although ${ }^{40} \mathrm{Ar} /{ }^{39} \mathrm{Ar}$ data from phenocrystic phlogopite from the dyke gave an age of $686 \pm 9 \mathrm{Ma}$. The lamprophyre dyke was recently discovered in one of the Kongsberg silver mines at Vinoren, Norway. Whole rock geochemistry, geochronological and mineralogical data from the ultramafic lamprophyre dyke are presented aiming to elucidate its origin and possible geodynamic setting. From the whole-rock composition of the Vinoren dyke, the rock could be recognized as transitional between carbonatite and kimberlite-II (orangeite). From its diagnostic mineralogy, the rock is classified as aillikite. The compositions and xenocrystic nature of several of the major and accessory minerals from the Vinoren aillikite are characteristic for diamondiferous rocks (kimberlites/lamproites/UML): Phlogopite with kinoshitalite-rich rims, chromite-spinel-ulvöspinel series, Mg- and Mn-rich ilmenites, rutile and lucasite-(Ce). We suggest that the aillikite melt formed during partial melting of a MARID (mica-amphibole-rutile-ilmenite-diopside)-like source under $\mathrm{CO}_{2}$ fluxing. The pre-rifting geodynamic setting of the Vinoren aillikite before the Rodinia supercontinent breakup suggests a relatively thick SCLM (Subcontinental Lithospheric Mantle) during this stage and might indicate a diamond-bearing source for the parental melt. This is in contrast to the about 100 Ma younger Fen complex, which were derived from a thin SCLM.
\end{abstract}

Keywords: aillikite; phlogopite; carbonate; spinel; ilmenite; titanite; diamond; Vinoren; Southern Norway

\section{Introduction}

Although ultramafic lamprophyres (UML) are volumetrically insignificant rocks, they may play a crucial role in the understanding of deep (mantle) melting events. UML form dyke swarms and rarely pipes commonly associated with continental extension, commencing during the initial stages of continental rifts evolution. UML often occurs together with alkaline mafic-ultramafic and carbonatitic 
intrusive complexes [1]. UML are classified as melanocratic rocks with abundant olivine and phlogopite macrocrysts and/or phenocrysts and can be subdivided into three rock types depending on a third essential mineral [2]. (1) Alnöits are melilite-bearing UML; (2) aillikites contain primary carbonate; and (3) damtjernites are nepheline- and/or alkali feldspar-bearing. Clinopyroxene and/or richteritic amphibole might be present in all three types, whereas spinel, ilmenite, rutile, perovskite, Ti-rich garnet, titanite, apatite are typical minor and accessory phases. UML show similarities to other volatile-rich rocks, such as kimberlites, lamproites and silicocarbonatites in terms of the occurrences and mineralogy. Nevertheless, some compositional differences between the rock types and their distinctly different geodynamic settings (rift-related for UML and stable cratonic for kimberlites and lamproites) suggest that they have different magma sources and petrogeneses. Similar to kimberlites and lamproites, UML may contain diamonds [3-7], indicating that the depth of magma generation for UML can be in excess of $130 \mathrm{~km}$.

During the period 750-600 Ma ago, the fragmentation of the supercontinent Rodinia was accompanied by voluminous continental and rift-related magmatism in both the North Atlantic Craton (NAC) and Baltica. Examples are ultramafic lamprophyres and carbonatites in NE Canada (Abloviak, Torngat of 600-580 Ma age, Aillik Bay-595-570 Ma, Saglek—570 Ma, Hebron—606 Ma, Eclipse Harbour-578 Ma, Killinek Island-576 Ma) and western Greenland (Sisimiut-Sarfartoq-Maniitsoq-610-550 Ma), as well as the carbonatite-ultramafic complexes (the Fen complex, Southern Norway-580 Ma) and kimberlites (eastern Finland-600-550 Ma) in Baltica. Several of the rocks that were emplaced during this event originated from diamond-bearing mantle depths, i.e., the Abloviak UML, northern Labrador, Canada [5,7], Sarfartoq kimberlite and UML, West Greenland [8] and the Kaavi-Kuopio kimberlites, Finland [9,10].

In this paper, the mineralogy, whole rock compositional data and the age of the recently discovered Vinoren UML dyke within the Kongsberg silver district, Kongsberg lithotectonic unit, Southern Norway, are presented. Based on the new data, the origin of the dyke and the geodynamic implications of the discovery will be discussed.

\section{Geological Setting}

The major part of the crust in Southern Norway is built up of Paleo- to Mesoproterozoic rocks that underwent multiphase reworking along the Fennoscandian margin during the Sveconorwegian Orogeny, between 1140 and $920 \mathrm{Ma}$ ago [11-13]. This orogeny was one of several orogenic events worldwide that resulted in the formation of the supercontinent Rodinia, and it has been inferred to result from the collision between proto-Baltica and Amazonia (e.g., [14-17]). However, an accretionary and non-collisional model for the formation of the Sveconorwegian Orogeny has also been proposed $[18,19]$. The orogenic belt has been sub-divided in five orogen-parallel lithotectonic units, which are separated by major Sveconorwegian shear zones: The Eastern Segment, Idefjorden, Kongsberg, Bamble and Telemarkia units [20].

The Kongsberg silver district is situated within the Kongsberg lithotectonic unit and includes a variety of gneisses (1600-1400 Ma) and granitoids (1171-1146 Ma) [17,21]. The silver district is characterized by subvertical zones enriched in sulfides (predominantly pyrite and pyrrhotite), inferred to be of hydrothermal origin. These zones, which are called fahlbands (e.g., [22,23]), are up to $900 \mathrm{~m}$ wide and subparallel to the foliation of the surrounding lithologies. The fahlbands and the older lithologies are crosscut by E-W trending dolerite dikes, quartz veins and silver bearing calcite veins of Permian age [24-26]. Already in the early days, the miners realized that the silver mineralizations occur almost exclusively at the intersections of the calcite veins and the fahlbands (e.g., [27]). Neumann [28] referred to the mineralized veins as calcite-nickel-cobalt-arsenide-native silver veins. The veins vary from a few millimeters up to $0.5 \mathrm{~m}$ in thickness, although up to several meters thick zones have been observed [28]. In a recent study of the silver mineralizations, Kotková et al. [29] gave an update of the paragenetic sequence presented by Neumann [28]. 
The UML dyke reported here occurs in the Klausstollen adit, adjacent to the Ringnesgangen underground silver mine, S. Vinoren, which is located in the northernmost part of the Kongsberg silver district (Figure 1). The dyke strikes toward NE with a dip of approximately $35^{\circ}$ toward NW (Figure 1 ). The dyke, which is about $50 \mathrm{~cm}$ thick, is fractured and tectonized; however, significant parts appears to be undeformed (Figure 2a). In places, the contact between the dyke and the host-rock appears as an undeformed and sharp intrusive contact. Some of the fractures within the dyke are filled with calcite.

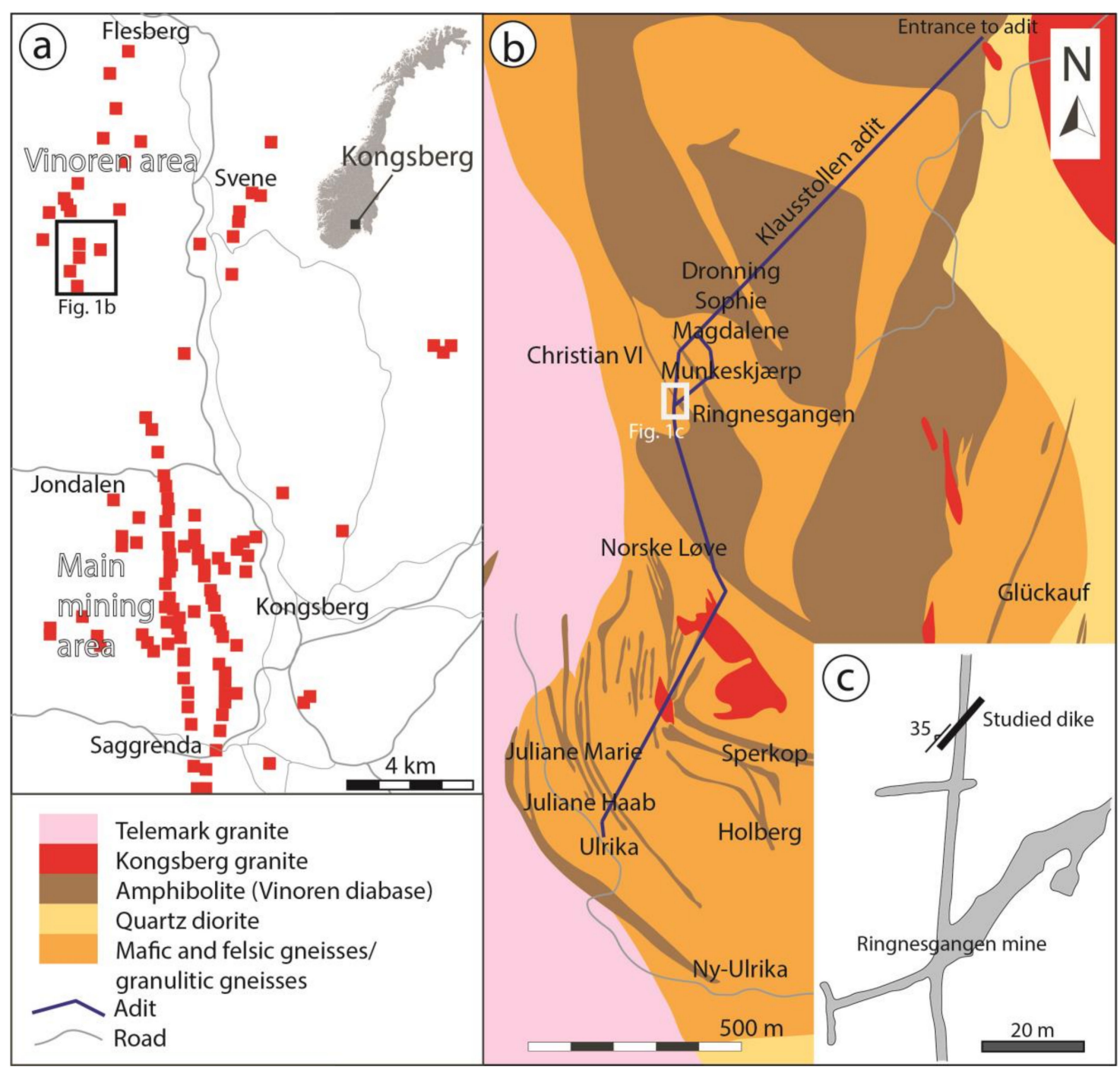

Figure 1. (a) Overview map showing the occurrences of silver mines in the Kongsberg silver district. Black rectangle shows location of (b). (b) Simplified geological map of the central part of the Vinoren area. White rectangle shows the location of (c). (c) Sketch showing the occurrence of the studied dyke in the Klausstollen adit, adjacent to the Ringnesgangen underground silver mine. 


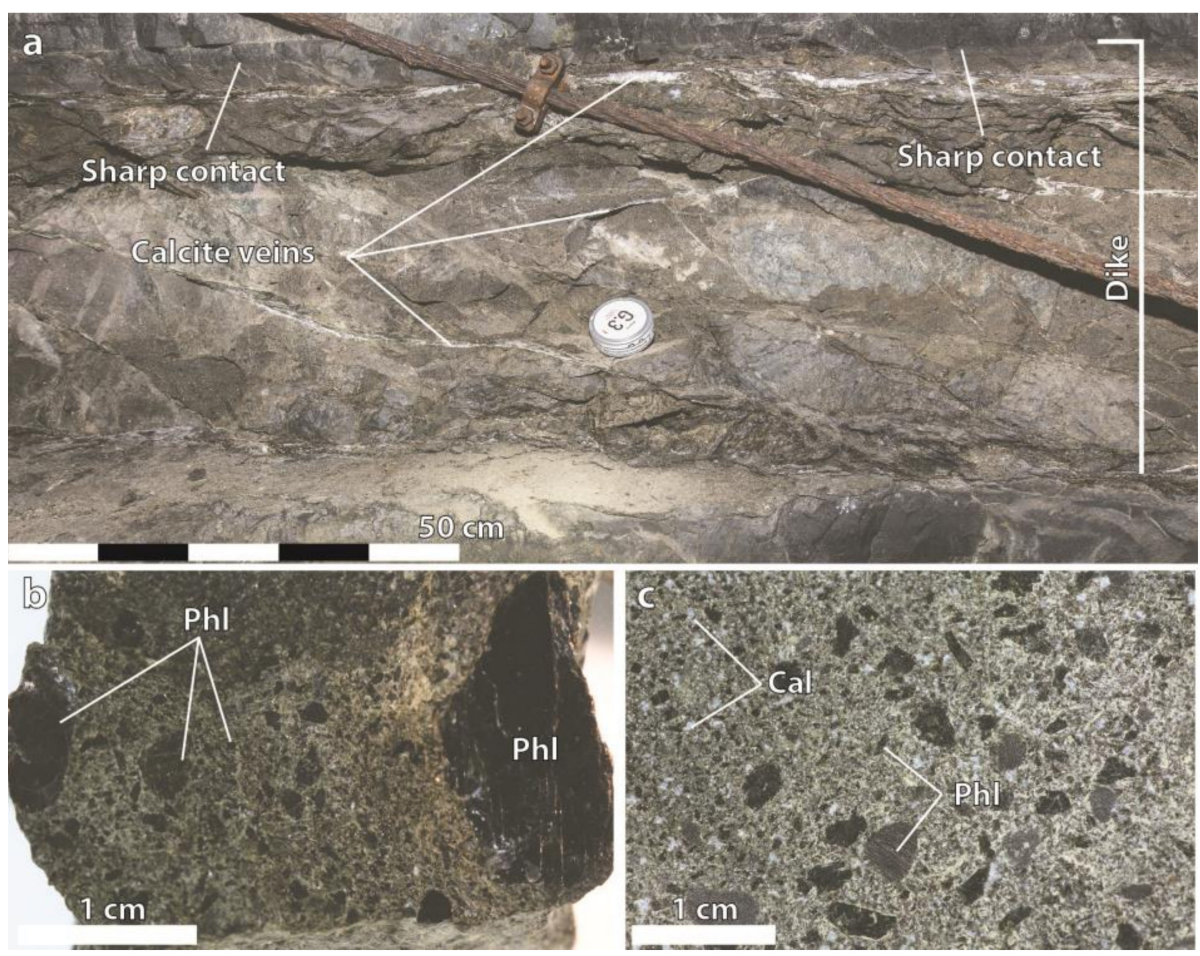

Figure 2. (a) Photo showing the contact relationships between the studied dyke and the host rock. Note the calcite veins crosscutting the dyke. (b) Hand specimen showing phlogopite phenocrysts up to $1 \mathrm{~cm}$ in diameter in a fine-grained groundmass. (c) Hand specimen showing phlogopite phenocrysts up to $4 \mathrm{~mm}$ in diameter and calcite crystals up to $1 \mathrm{~mm}$ in diameter in a fine-grained groundmass.

\section{Analytical Methods}

\subsection{Mineral Analyses}

Chemical analyses of minerals from the Vinoren dyke were carried out using a Cameca MS-46 electron microprobe analyzer (EMPA) (CAMECA, Gennevilliers, France) at the Geological Institute, Kola Science Center, Apatity, Russia. The instrument was operated in a wavelength-dispersive mode at the following conditions: Acceleration voltage $22 \mathrm{kV}$, beam current $30-40 \mathrm{nA}, 50 \mathrm{sec}$ counting time. The following calibrating materials (and analytical lines) were used: Wollastonite ( $\mathrm{Si} K \alpha, \mathrm{CaK} \alpha$ ), hematite $(\mathrm{Fe} K \alpha)$, apatite $(\mathrm{PK} \alpha)$, lorenzenite $(\mathrm{NaK} \alpha)$, thorite $(\mathrm{ThM} \alpha), \mathrm{MnCO}_{3}(\mathrm{MnK} \alpha), \mathrm{Y}_{3} \mathrm{Al}_{5} \mathrm{O}_{12}(\mathrm{YL} \alpha),(\mathrm{La}, \mathrm{Ce}) \mathrm{S}$ $(\mathrm{La} L \alpha), \mathrm{CeS}(\mathrm{Ce} L \alpha), \mathrm{Pr}_{3} \mathrm{Al}_{5} \mathrm{O}_{12}\left(\operatorname{Pr} L \beta_{1}\right), \mathrm{LiNd}\left(\mathrm{MoO}_{4}\right)_{2}(\mathrm{Nd} L \alpha), \mathrm{SmFeO}_{3}(\mathrm{Sm} L \alpha), \mathrm{EuFeO}_{3}(\mathrm{Eu} L \alpha), \mathrm{GdS}$ $(\mathrm{Gd} L \alpha), \mathrm{TbPO}_{4}(\mathrm{~Tb} L \alpha), \mathrm{Dy}_{3} \mathrm{Al}_{5} \mathrm{O}_{12}(\mathrm{Dy} L \alpha), \mathrm{Ho}_{3} \mathrm{Ga}_{5} \mathrm{O}_{12}\left(\mathrm{Ho} L \beta_{1}\right), \mathrm{ErPO}_{4}(\mathrm{Er} L \alpha), \mathrm{Tm}_{3} \mathrm{Al}_{5} \mathrm{O}_{12}(\mathrm{Tm} L \alpha)$, $\mathrm{Yb}_{3} \mathrm{Al}_{5} \mathrm{O}_{12}(\mathrm{YbL} \alpha)$, and $\mathrm{Y}_{2.8} \mathrm{Lu}_{0.2} \mathrm{Al}_{5} \mathrm{O}_{12}(\mathrm{Lu} L \alpha)$. Detection limits for $\mathrm{Fe}, \mathrm{Mn}$ are $0.01 \% ; \mathrm{Si}, \mathrm{Al}, \mathrm{Cl}, \mathrm{Ca}, \mathrm{K}$, $\mathrm{Cl}-0.02 \% ; \mathrm{P}, \mathrm{Na}, \mathrm{Y}, \mathrm{Sr}, \mathrm{La}, \mathrm{Ce}, \mathrm{Nd}-0.03 \% ; \mathrm{Ba}-0.05 \% ; \mathrm{Nb}, \mathrm{Zr}-0.1 \%$.

Accessory mineral identification and qualitative composition of grains and mineral inclusions less than 20-30 $\mu \mathrm{m}$ was performed using a LEO-1450 SEM (scanning electron microscope) (Carl Zeiss AG, Oberkochen, Germany) equipped with XFlash-5010 Bruker Nano GmbH EDS (energy-dispersive Xray spectroscopy). The system was operated at $20 \mathrm{kV}$ acceleration voltage, $0.5 \mathrm{nA}$ beam current, with $200 \mathrm{~s}$ accumulation time.

Materials from minerals forming possible pseudomorphs after olivine close to points analyzed by microprobe were examined by the X-ray diffraction (XRD) method (Debye-Scherer) by means of an URS-1 (Bourevestnik JSC, Saint-Petersburg, Russia) operated at $40 \mathrm{kV}$ and $16 \mathrm{~mA}$ with RKU-114.7 mm camera and $\mathrm{Fe} K \alpha$-radiation. 


\subsection{Whole Rock Analyses}

Whole rock compositions were obtained at the Kola Science Center in Apatity, Russia. Most of the major elements were determined by atomic absorption spectrophotometry; $\mathrm{TiO}_{2}$ by colorimetry; $\mathrm{K}_{2} \mathrm{O}$, $\mathrm{Na}_{2} \mathrm{O}, \mathrm{Cu}, \mathrm{Ni}, \mathrm{Co}, \mathrm{Cr}, \mathrm{V}, \mathrm{Rb}, \mathrm{Cs}$, and $\mathrm{Li}$ by flame photometry; $\mathrm{FeO}$ and $\mathrm{CO}_{2}$ by titration (volumetric analysis); and $\mathrm{F}$ and $\mathrm{Cl}$ by potentiometry using an ion-selective electrode (for the full description of the methods, see [30]).

\section{3. ${ }^{40} \mathrm{Ar}{ }^{\beta 9} \mathrm{Ar}$ Analyses}

Fragment of phlogopite with diameter about $1 \mathrm{~mm}$ was hand-picked from one phenocrystic sample of the dyke rock, cleaned by ultrasonic bath and dried up at $40{ }^{\circ} \mathrm{C}$. The mineral fragment was in cadmium foil. The grain was placed in a capsule made of $99.999 \%$ aluminum. The sample was irradiated for neutron activation at the CLICIT (cadmium-lined-in-core irradiation tube) facility at the Oregon State TRIGA reactor (OSTR), Oregon State University, Oregon, USA. To obtain the degree of neutron activation (J), the neutron flux monitoring mineral Fish Canyon Tuff sanidine (27.5 Ma [31,32]) was used. To correct possible interference of Ar isotopes produced by the reaction of $\mathrm{K}$ and $\mathrm{Ca}$, crystals of $\mathrm{K}_{2} \mathrm{SO}_{4}$ and $\mathrm{CaF}_{2}$ were irradiated separately. Irradiation time was $4 \mathrm{~h}$, and the fast neutron flux was $2.47 \times 10^{13} \mathrm{n} / \mathrm{cm}^{2} / \mathrm{s}$. After irradiation, the sample was cooled down for one month and transported to the Ar/Ar laboratory at the University of Potsdam, Germany. The sample was analyzed with a Gantry Dual Wave laser ablation system by the stepwise heating method until total melting. The system work with a $50 \mathrm{~W} \mathrm{CO}_{2}$ laser (wavelength of $10.6 \mu \mathrm{m}$ ), using a defocused continuous laser beam with a diameter of maximum $1500 \mu \mathrm{m}$ during $1 \mathrm{~min}$ for heating and gas extraction. The released sample gas was exposed to the SAES getters and cold stainless trap cooled at $-90^{\circ} \mathrm{C}$ through the ethanol by electric cooler in order to purify the sample gas to pure Ar for $10 \mathrm{~min}$ in a closed ultra-high vacuum purification line. The pure argon gas was analyzed by a Micromass 5400 noble gas mass spectrometer with high sensitivity and ultra-low background. The spectrometer operates with an electron multiplier for very small amounts of gas. During the measurements, blanks were measured every third step. The software Mass Spec, designed by Dr. Alan Deino of Berkeley Geochonology Center, Berkeley, CA, USA was used for processing the data. The recommended atmospheric ${ }^{40} \mathrm{Ar} /{ }^{36} \mathrm{Ar}$ ratio of 295.5 and the decay constants for $\lambda\left({ }^{40} \mathrm{~K}_{\beta}{ }^{-}\right)=4.962 \times 10^{-10} / \mathrm{yr}$ and $\lambda\left({ }^{40} \mathrm{~K}_{\mathrm{e}}\right)=0.581 \times 10^{-10} / \mathrm{yr}$ were used [33]. Used interference correction parameters are: $\left({ }^{36} \mathrm{Ar} /{ }^{37} \mathrm{Ar}\right)_{\mathrm{Ca}}=2.73 \pm 0.032 \times 10^{-4},\left({ }^{39} \mathrm{Ar} /{ }^{37} \mathrm{Ar}\right)_{\mathrm{Ca}}=6.638$ $\pm 0.263 \times 10^{-4},\left({ }^{40} \mathrm{Ar} /{ }^{39} \mathrm{Ar}\right)_{\mathrm{K}}=50.966 \pm 24.353 \times 10^{-4}$, and $\left({ }^{38} \mathrm{Ar} /{ }^{39} \mathrm{Ar}\right)_{\mathrm{K}}=1.1816 \pm 0.00266 \times 10^{-2}$. All errors correspond to 1 sigma error.

\section{Results and Primary Interpretation}

\subsection{Petrography and Mineral Compositions}

In hand specimen, the UML rock is massive and characterized by anhedral phenocrysts of phlogopite (up to $1 \mathrm{~cm}$ in diameter) and calcite (up to $1 \mathrm{~mm}$ in diameter), and rounded aggregates of a serpentine-like mineral (up to $3 \mathrm{~mm}$ in diameter) in a fine-grained, grey groundmass (Figure $2 \mathrm{~b}, \mathrm{c}$ ). The groundmass (Figure 3a) is composed of phlogopite (20-25 vol.\%), carbonate (20-25 vol.\%), serpentine-like mineral (about $40 \mathrm{vol} . \%$ ) and titanite (5-6 vol.\%). Minor and accessory minerals are apatite (2-3 vol.\%), magnetite (2-3 vol.\%), rutile (1-2 vol.\%), quartz (1-2 vol.\%), lucasite-(Ce) $\left[\mathrm{CeTi}_{2}(\mathrm{O}, \mathrm{OH})_{6}\right]$, ilmenite, garnet, spinel, zircon, barite, strontianite, celestine, godlevskite $\left[(\mathrm{Ni}, \mathrm{Fe})_{9} \mathrm{~S}_{8}\right]$, galena, sphalerite, pyrite, chalcopyrite, and pentlandite.

The carbonate in the groundmass is represented by almost pure calcite with $<0.1 \mathrm{wt} . \% \mathrm{MgO}$ (Table 1). We infer that the mineral is primary as it forms triple-junction boundaries between intergrown grains (Figure 3f). Secondary calcite occurs in aggregates with serpentine-like minerals and is characterized by high SrO content (up to $2 \mathrm{wt} . \%$ ).

Phlogopite occurs both as phenocrysts and as grains up to $1 \mathrm{~mm}$ in the groundmass (Table 2). The phenocrystic phlogopite is homogenous, whereas two types of chemical zonation can be observed 
in the groundmass phlogopite. In back-scatter electron (BSE) images, the first type of zonation is characterized by a dark core and brighter rim of phlogopite (Figure 3e). The bright rim typically shows higher $\mathrm{BaO}$ than the core. The second type of zonation is represented by a few $\mu \mathrm{m}$ thick bright rims in BSE images (Figures 3e and 4), reflecting elevated $\mathrm{FeO}$ and lower $\mathrm{Al}_{2} \mathrm{O}_{3}$ and $\mathrm{MgO}$ in the thin rims. The groundmass phlogopites are sometimes bent suggesting that the mineral already had formed when the magma was emplaced as a crystal mush.

The serpentine-like aggregates consist of a mixture of a mineral that is closer in composition to saponite than serpentine, and minor talc (Table 3). The presence of saponite has been confirmed by XRD analysis. The formation of saponite after olivine and serpentine during low-temperature hydrothermal alteration has been reported from some kimberlite occurrences (e.g., in the Arkhangelsk province, [34]).

Spinel occurs as 20-30 $\mu \mathrm{m}$ anhedral, often resorbed grains associated with rutile and lucasite-(Ce), all included in titanite (Figure 5a-d). Spinel grains often show reaction rims composed of an aggregate of calcite and saponite along the contact to the hosting titanite (Figure $5 b, d$ ). Based on the morphology and textural relationships of spinel, it is inferred that it is xenocrystic. The mineral is characterized by variable contents of $\mathrm{Cr}_{2} \mathrm{O}_{3}$ (13-27 wt.\%), FeO (50-66 wt.\%), MgO (0-7.75 wt.\%), $\mathrm{TiO}_{2}(7-11$ wt.\%) and $\mathrm{Al}_{2} \mathrm{O}_{3}$ (4.9-6.8 wt.\%) and represents presumably chromite-spinel- ulvöspinel/titanomagnetite solid solutions (Table 4; full dataset is in Supplementary Table S1). In BSE images, spinel is often zoned with darker central parts containing higher $\mathrm{Cr}_{2} \mathrm{O}_{3}, \mathrm{MgO}$ and $\mathrm{Al}_{2} \mathrm{O}_{3}$ and lower $\mathrm{FeO}, \mathrm{MnO}$ and $\mathrm{TiO}_{2}$ compared to the outer parts of the grains. The average composition of the inner parts of the zoned spinel gives the formula $\left(\mathrm{Mg}_{0.44} \mathrm{Fe}_{0.31} \mathrm{Ti}_{0.21} \mathrm{Mn}_{0 \cdot 02} \mathrm{Ca}_{0.01} \mathrm{Ni}_{0.01} \mathrm{Zn}_{0.01}\right)_{0.99}\left(\mathrm{Fe}_{0.9} \mathrm{Cr}_{0.77} \mathrm{Al}_{0.34}\right)_{2.01} \mathrm{O}_{4}$ which mainly corresponds to the spinel-chromite-ulvöspinel solid solution. The outer parts give the formula $\left(\mathrm{Fe}_{0.50} \mathrm{Ti}_{0.27} \mathrm{Mn}_{0.12} \mathrm{Zn}_{0.04} \mathrm{Mg}_{0.02} \mathrm{Ca}_{0.02} \mathrm{Ni}_{0.01}\right)_{0.98}\left(\mathrm{Fe}_{1.30} \mathrm{Cr}_{0.47} \mathrm{Al}_{0.23}\right)_{2} \mathrm{O}_{4}$ corresponding to the magnetiteulvöspinel-manganchromite solid solution. Overall, the spinel studied here is similar to spinel from UML (i.e., Torngat occurrence, [35]) and differs from kimberlite and lamproite spinels by lower $\mathrm{Cr}_{2} \mathrm{O}_{3}$ and elevated $\mathrm{TiO}_{2}[36]$.

Titanite occurs as euhedral and subhedral grains, up to $100 \mu \mathrm{m}$ in diameter (Figure 5). The mineral contains abundant inclusions of rutile, suggesting that titanite formed during breakdown of rutile at high activities of $\mathrm{Si}$ and $\mathrm{Ca}$. Titanite is characterized by elevated $\mathrm{Al}_{2} \mathrm{O}_{3}(0.5-0.8$ wt. \%) and $\mathrm{FeO}$ (4.1-4.8 wt.\%) (Table 5). The MgO content of titanite varies in the range $0.2-1.3 \mathrm{wt} . \%$, while $\mathrm{La}_{2} \mathrm{O}_{3}+$ $\mathrm{Ce}_{2} \mathrm{O}_{3}$ shows concentrations in the range $0.3-0.5 \mathrm{wt} . \%$.

Ilmenite is present as the two solid solution series geikielite-ilmenite and ilmenite-pyrophanite. The first one occurs as ca. $200 \mu \mathrm{m}$ rounded resorbed grains with titanite rims (Figure 5e). The composition of the grains varies from core to rim mainly in $\mathrm{MgO}$ (from 12 to $2 \mathrm{wt}$ \%), $\mathrm{FeO}$ (from 31 to $42 \mathrm{wt} . \%$ ) and $\mathrm{MnO}$ (from 0.4 to $3.9 \mathrm{wt} \%$ ) (Table 5). The mineral is characterized by the presence of $\mathrm{Al}_{2} \mathrm{O}_{3}$ (0.44-0.57 wt.\%), $\mathrm{NiO}$ (0.12 wt.\%), $\mathrm{Cr}_{2} \mathrm{O}_{3}$ (up to 0.09 wt.\%) and $\mathrm{CaO}$ (up to 0.13 wt.\%). Ilmenite of similar Mg-rich composition is an indicative mineral for diamondiferous kimberlites. The compositional zonation revealed for ilmenite from the studied dyke is similar to that from Torngat UML. Ilmenite corresponding to the ilmenite-pyrophanite series (up to $16 \mathrm{wt}$ \% of MnO) occurs as single 10-20 $\mu \mathrm{m}$ grains included in titanite (Figure 5f). Ilmenite compositions like this are characteristic for carbonatites.

Rutile is a relatively abundant accessory mineral, found in titanite in association with lucasite-(Ce) (Figure $5 b, g, h)$. The replacement of rutile by titanite apparently took place during a late-magmatic carbonatization stage with high Ca- and REE-activities. Rutile is characterized by a moderate $\mathrm{Nb}_{2} \mathrm{O}_{5}$ content (0.4-0.6 wt.\% (Table 5)) that is different from typical Nb-rich kimberlitic rutile. The associated lucasite-(Ce) belongs to the same stage and occurs as needles included in titanite. Lucasite-(Ce) is a characteristic mineral of diamondiferous lamproite, e.g., from Argyle, Western Australia [37]. Vinoren lucasite-(Ce) differs from the lamproitic mineral by elevated $\mathrm{CaO}$ (3.3-5.5 wt.\%, Table 5).

Garnet is a secondary minor mineral formed as bud-shaped grains associated with saponite and in interstices between grains of phlogopite (Figure 6e,f). EMPA data (Table 3) indicates that the 
mineral is hydroandradite $\left[\mathrm{Ca}_{3} \mathrm{Fe}^{3+}{ }_{2}\left(\mathrm{SiO}_{4}\right)_{3-\mathrm{x}}(\mathrm{OH})_{4 \mathrm{x}}\right]$ with low to moderate $\mathrm{TiO}_{2}$ content $(0.3-1.2 \mathrm{wt} . \%)$, in contrast to the Ti-rich garnets that is characteristic for UML.

Apatite forms elongated and needle-shaped crystals up to $250 \mu \mathrm{m}$ long (Figure 6d). The mineral classifies as fluorapatite, but it contains significant amount of other volatile elements (F: 1.5-1.7 wt.\%; Cl: $0.07-0.09$ wt.\%; $\mathrm{SO}_{3}$ : 0.16-0.25 wt.\%).
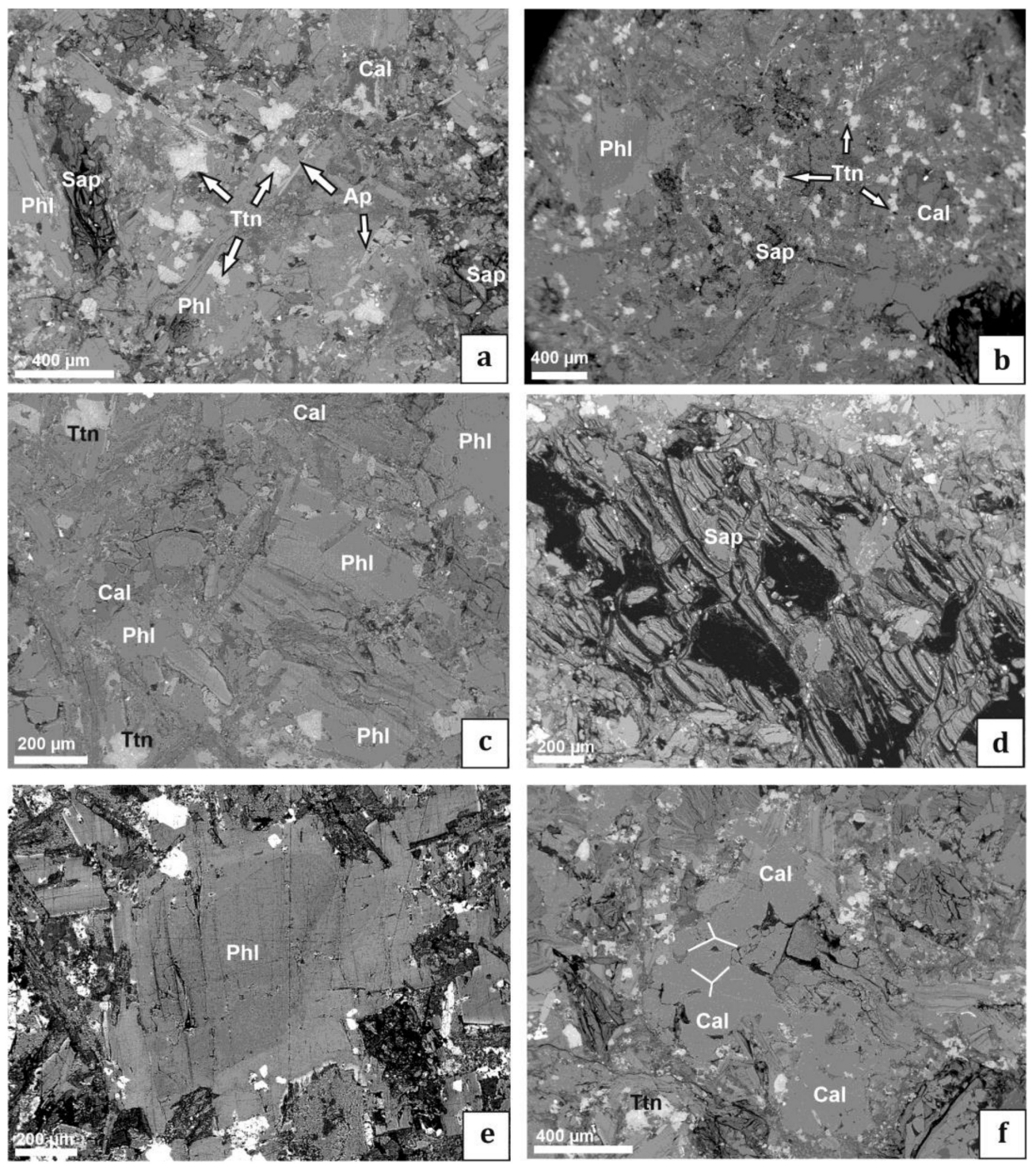

Figure 3. Back-scattered electron (BSE) images showing textural relationships between different minerals and the morphology of the major minerals: (a) Phlogopite and saponite phenocrysts in groundmass of phlogopite, calcite, titanite and apatite; (b) phlogopite phenocrysts and calcite vugs in groundmass of phlogopite, calcite and titanite; (c) euhedral and subhedral phlogopite, anhedral calcite and subhedral titanite from the groundmass; (d) typical oval-shaped aggregate of saponite+/-talc (possibly after olivine), black spots are the holes; (e) zoned groundmass phlogopite with BSE-higher Ba-rich thick rims and BSE-higher Fe-rich thin rims; (f) triple junctions in cluster of calcite grains. Mineral abbreviations: $\mathrm{Ap}=$ apatite, $\mathrm{Cal}=$ calcite, $\mathrm{Phl}=$ phlogopite, $\mathrm{Sap}=$ saponite, $\mathrm{Ttn}=$ titanite.

Zircon occurs as needles of about $20 \mu \mathrm{m}$ long, assembled in subparallel aggregates (Figure 6a,b). The skeletal form of zircon indicates rapid growth of the mineral.

A Ni-Fe-S mineral phase with the composition 30.8 wt.\% S, 38.9 wt.\% Ni, $27.1 \mathrm{wt} . \% \mathrm{Fe}$ and $3.1 \mathrm{wt} . \% \mathrm{Co}$ (possibly godlevskite: $(\mathrm{Ni}, \mathrm{Fe})_{9} \mathrm{~S}_{8}$ ), which occurs as numerous rounded grains of $1-2 \mu \mathrm{m}$ in diameter in the saponite-talc aggregates (Figure 6c), is inferred to be an alteration product after olivine. 
Secondary quartz occurring in the saponite-talc aggregates is also inferred to be an alteration product after olivine. Barite and strontianite form anhedral grains, 1-3 $\mu \mathrm{m}$ in diameter, occur as inclusions in calcite. Other accessory phases that were observed (pyrite, galena, chalcopyrite, sphalerite, pentlandite, and celestine) in couple with other sulfides and sulfates indicate a relatively high $\mathrm{S}$ activity during the formation of the studied dyke.
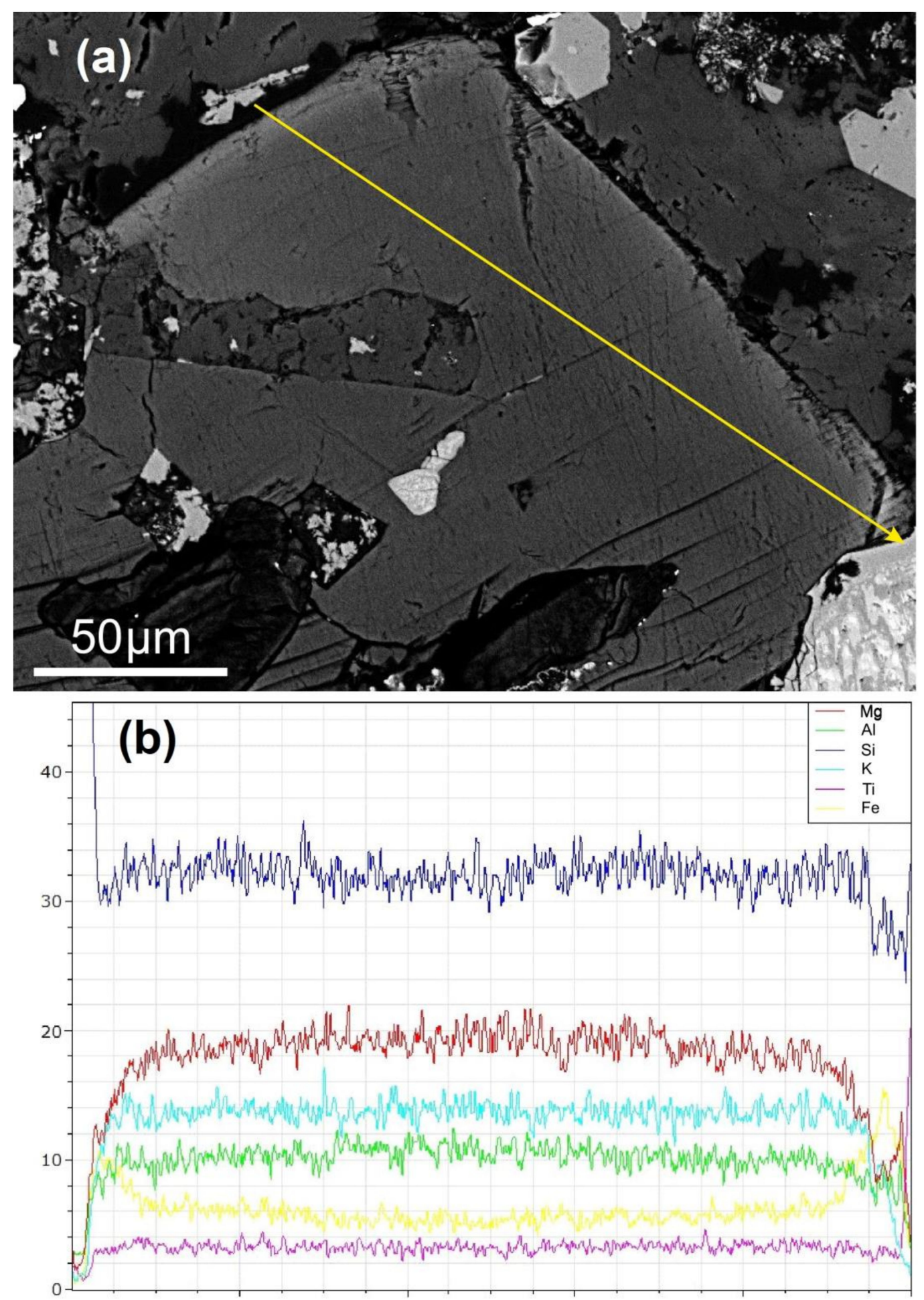

Figure 4. Compositional variations across zoned groundmass phlogopite (a)-BSE image with profile location; (b) -relative characteristic X-ray intensities for selected elements, showing the relatively lower $\mathrm{Mg}, \mathrm{Al}$ and higher Fe concentrations in rims). 

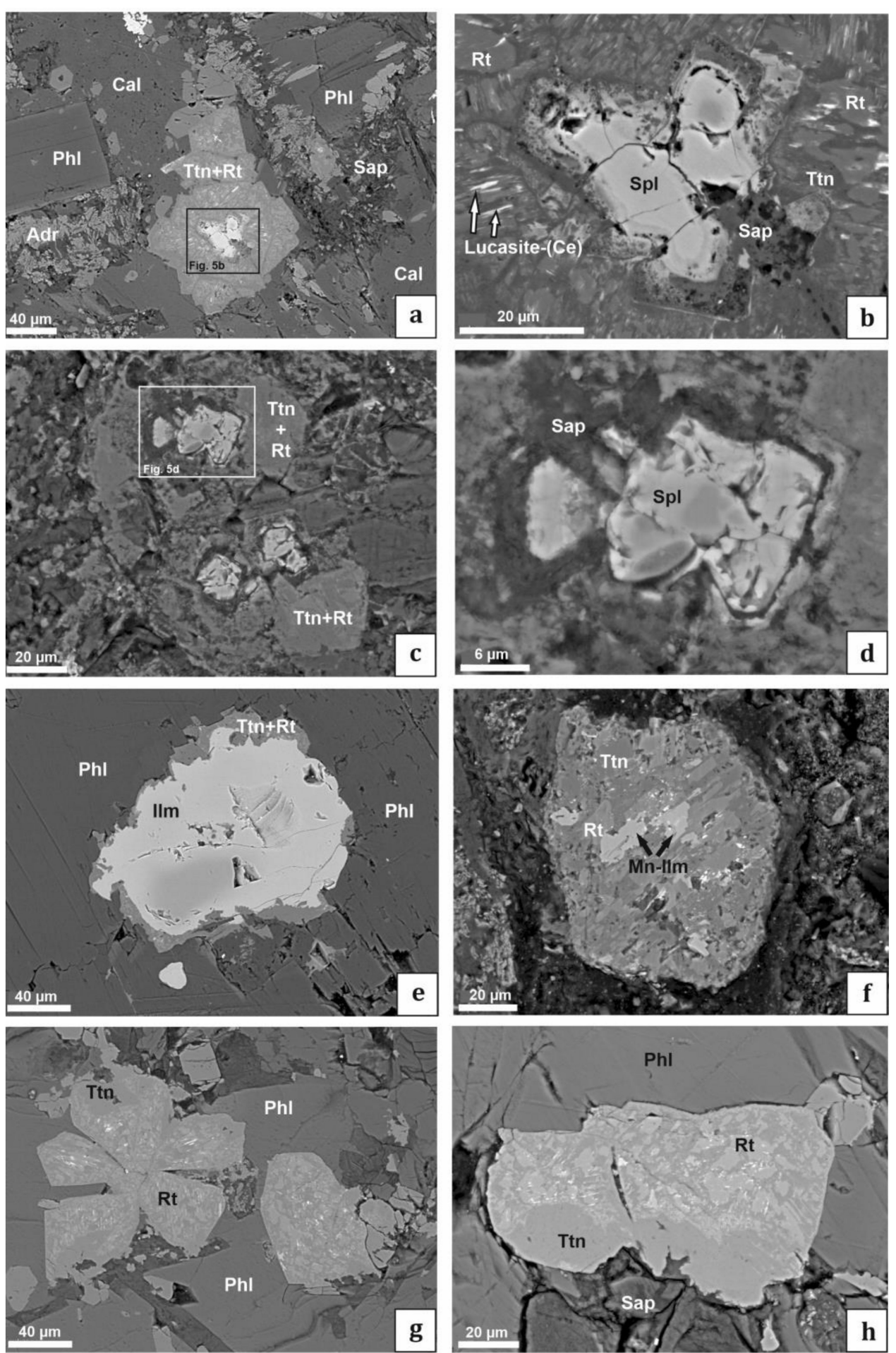

Figure 5. BSE images showing the textural relationships, morphology and internal textures of spinel, ilmenite, titanite and rutile: (a) Subhedral titanite grain with inclusions of rutile (light-gray), lucasite-(Ce) (brightest needles) and corroded spinel grain; (b) typical corroded spinel grain with reaction rim composed mainly of saponite; (c) several spinel grains in the center of titanite-rutile aggregate; (d) zoned spinel grain with high-Mg and low-Fe cores; (e) zoned corroded grain of ilmenite with rims of titanite-rutile intergrowths and core enriched in $\mathrm{Mg}$; (f) inclusions of Mn-rich ilmenite (light gray) and rutile (gray) in titanite (dark gray); (g) morphology of titanite-rutile-lucasite-(Ce) intergrowths and (h) irregular distribution of rutile (light gray) and lucasite-(Ce) (bright needles) in titanite. Mineral abbreviations as in Figure 3; in addition: Adr = hydroandradite, Ilm = ilmenite, $\mathrm{Rt}=$ rutile, Spl = spinel. 

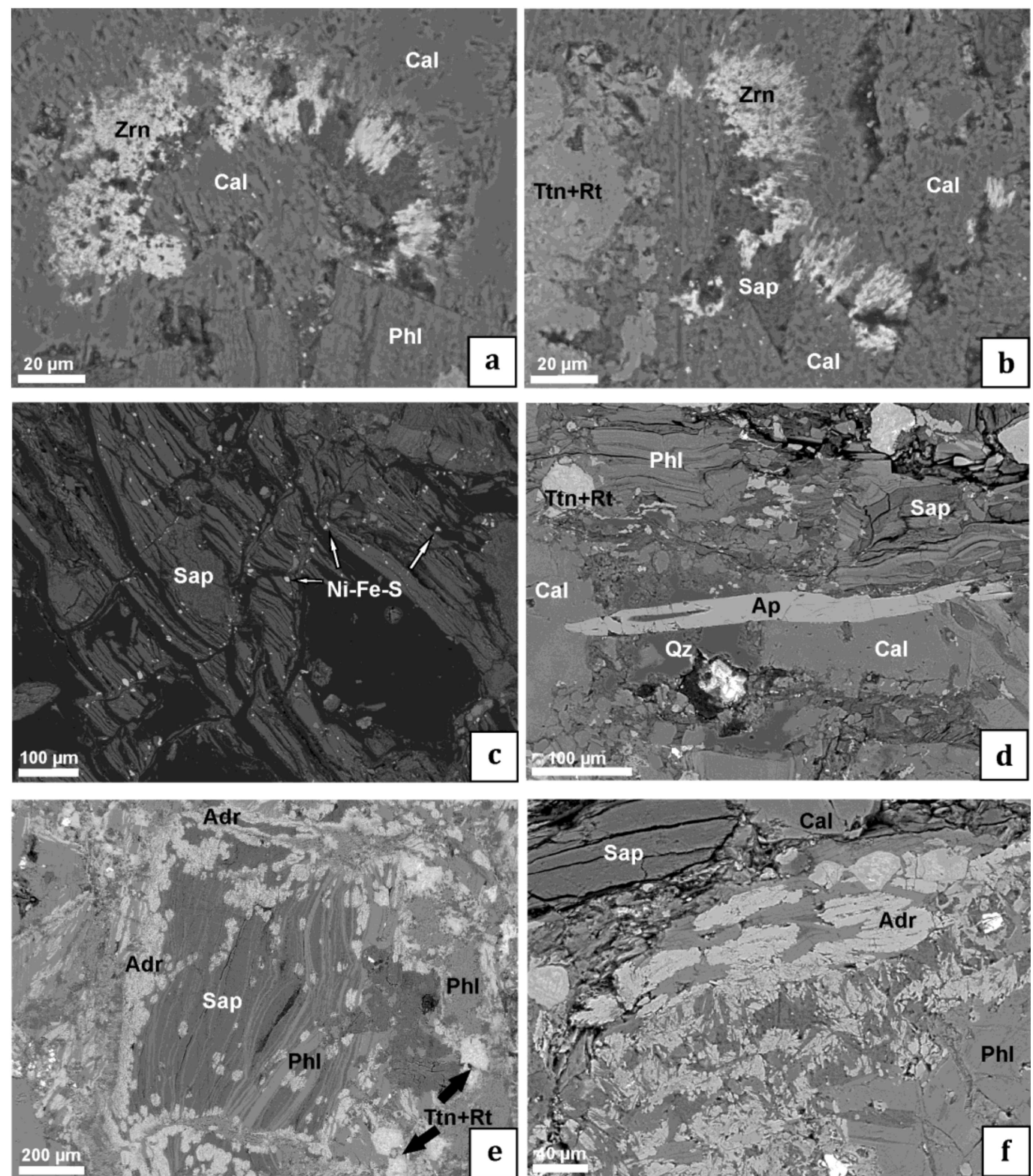

Figure 6. BSE images showing the morphology and textural relationships of minor, accessory and secondary minerals: (a,b) "Skeletal” zircon (possibly due to rapid growth); (c) Numerous grains of Ni-Fe sulfide (Ni > Fe, possibly godlevskite) (bright) included in saponite aggregate; (d) typical morphology of apatite and bent groundmass phlogopite; (e,f) morphology of hydroandradite crystallized after phlogopite and saponite. Mineral abbreviations as in Figures 3 and 4; in addition: Zrn = zircon, $\mathrm{Ni}-\mathrm{Fe}-\mathrm{S}=\mathrm{Ni}-\mathrm{Fe}$ sulfide.

Table 1. Representative chemical compositions (wt.\%) and mineral formulae (apfu) of carbonate from the Vinoren aillikite.

\begin{tabular}{ccccccccc}
\hline Analysis \# & $\mathbf{2 c}$ & $\mathbf{2 e}$ & $\mathbf{2 - 6 a}$ & $\mathbf{2 - 7 a}$ & $\mathbf{2 - 7 - 1 a}$ & $\mathbf{2 - 8 a}$ & $\mathbf{3 - 1 a}$ & 4c \\
\hline $\mathrm{FeO}$ & 0.02 & 0.03 & 0.10 & $\mathrm{bdl}$ & 0.04 & bdl & bdl & bdl \\
$\mathrm{MnO}$ & 0.19 & 0.09 & $\mathrm{bdl}$ & $\mathrm{bdl}$ & bdl & 0.07 & bdl & bdl \\
$\mathrm{MgO}$ & 0.08 & 0.06 & 0.10 & $\mathrm{bdl}$ & bdl & bdl & bdl & bdl \\
$\mathrm{CaO}$ & 54.44 & 55.27 & 55.22 & 55.09 & 55.74 & 55.37 & 55.61 & 55.80 \\
$\mathrm{BaO}$ & $\mathrm{bdl}$ & $\mathrm{bdl}$ & $\mathrm{bdl}$ & $\mathrm{bdl}$ & bdl & bdl & bdl & bdl \\
$\mathrm{SrO}$ & 0.07 & $\mathrm{bdl}$ & 2.02 & 0.38 & bdl & 0.42 & bdl & bdl \\
Total & 54.80 & 55.45 & 57.44 & 55.47 & 55.78 & 55.86 & 55.61 & 55.80 \\
\hline
\end{tabular}


Table 1. Cont

\begin{tabular}{|c|c|c|c|c|c|c|c|c|}
\hline Analysis \# & $2 c$ & $2 e$ & $2-6 a$ & $2-7 a$ & 2-7-1a & $2-8 \mathbf{a}$ & 3-1a & $4 c$ \\
\hline \multicolumn{9}{|c|}{ Formulae based on $\Sigma$ cations = 1} \\
\hline $\mathrm{Fe}$ & & & 0.001 & & 0.001 & & & \\
\hline Mn & 0.003 & 0.001 & & & & 0.001 & & \\
\hline $\mathrm{Mg}$ & 0.002 & 0.002 & 0.002 & & & & & \\
\hline $\mathrm{Ca}$ & 0.994 & 0.997 & 0.977 & 0.996 & 0.999 & 0.995 & 1.000 & 1.000 \\
\hline Ва & & & & & & & & \\
\hline $\mathrm{Sr}$ & 0.001 & 0 & 0.019 & 0.004 & & 0.004 & & \\
\hline Total & 1.000 & 1.000 & 1.000 & 1.000 & 1.000 & 1.000 & 1.000 & 1.000 \\
\hline
\end{tabular}

Table 2. Representative chemical compositions (wt.\%) and mineral formulae (apfu) of mica from the Vinoren aillikite.

\begin{tabular}{|c|c|c|c|c|c|c|c|c|c|c|c|c|}
\hline Analysis \# & $1 a$ & $1 b$ & $2-2 a$ & $2-3 b$ & $2-3 c$ & $2-1 a$ & $2-1 b$ & $1-1 a$ & $1-2 a$ & $3-1 a$ & $1-1 a$ & $1-1 b$ \\
\hline & $\mathrm{P}$ & $\mathrm{P}$ & G & G & G & G & G & G & G & G & G & G \\
\hline & & & core & rim & rim & core & rim & rim & core & core & rim & core \\
\hline $\mathrm{SiO}_{2}$ & 38.44 & 37.64 & 36.92 & 37.34 & 37.02 & 38.20 & 38.19 & 38.82 & 36.86 & 39.81 & 35.15 & 36.30 \\
\hline $\mathrm{Al}_{2} \mathrm{O}_{3}$ & 12.29 & 12.45 & 13.13 & 11.02 & 13.66 & 12.53 & 9.27 & 13.47 & 14.12 & 12.14 & 14.70 & 13.02 \\
\hline $\mathrm{TiO}_{2}$ & 5.75 & 7.10 & 3.99 & 3.68 & 4.35 & 3.75 & 4.40 & 6.04 & 3.95 & 3.87 & 4.04 & 5.92 \\
\hline $\mathrm{FeO}$ & 9.12 & 9.84 & 8.23 & 8.99 & 7.54 & 8.07 & 22.96 & 8.78 & 6.89 & 7.82 & 7.12 & 8.63 \\
\hline $\mathrm{MnO}$ & bdl & 0.06 & 0.09 & 0.12 & 0.07 & 0.09 & 0.40 & 0.03 & 0.08 & 0.11 & 0.09 & bdl \\
\hline $\mathrm{MgO}$ & 16.77 & 17.42 & 19.38 & 18.65 & 19.45 & 19.88 & 11.62 & 18.56 & 21.43 & 21.76 & 21.83 & 18.89 \\
\hline $\mathrm{CaO}$ & 0.41 & bdl & bdl & bdl & bdl & 0.06 & 0.15 & 0.05 & 0.12 & 0.07 & 0.09 & 0.04 \\
\hline $\mathrm{Na}_{2} \mathrm{O}$ & 0.15 & 0.07 & 0.07 & 0.09 & 0.11 & 0.09 & bdl & 0.23 & 0.16 & 0.27 & 0.18 & 0.28 \\
\hline $\mathrm{K}_{2} \mathrm{O}$ & 13.27 & 12.16 & 12.50 & 12.85 & 12.51 & 12.12 & 10.50 & 10.27 & 9.93 & 10.27 & 12.84 & 13.36 \\
\hline $\mathrm{BaO}$ & 0.17 & 0.33 & 1.45 & 0.58 & 2.34 & 1.23 & 0.20 & na & na & na & 2.29 & 0.28 \\
\hline $\mathrm{NiO}$ & 0.07 & 0.09 & bdl & 0.06 & bdl & 1.04 & bdl & 0.05 & bdl & bdl & 0.04 & 0.07 \\
\hline $\mathrm{V}_{2} \mathrm{O}_{5}$ & bdl & bdl & bdl & bdl & bdl & bdl & 0.14 & bdl & bdl & bdl & bdl & 0.08 \\
\hline $\mathrm{SO}_{3}$ & na & na & na & na & na & na & na & 0.11 & bdl & bdl & bdl & 0.12 \\
\hline $\mathrm{Cl}$ & na & na & na & na & na & na & na & 0.03 & bdl & bdl & bdl & 0.05 \\
\hline Total & 96.44 & 97.15 & 95.74 & 93.38 & 97.05 & 97.05 & 97.84 & 96.44 & 93.53 & 96.12 & 98.36 & 96.86 \\
\hline \multicolumn{13}{|c|}{ Formulae based on $11 \mathrm{O}$} \\
\hline $\mathrm{Si}$ & 2.837 & 2.756 & 2.753 & 2.859 & 2.731 & 2.804 & 2.929 & 2.801 & 2.721 & 2.865 & 2.572 & 2.679 \\
\hline $\mathrm{Al}$ & 1.069 & 1.074 & 1.154 & 0.994 & 1.188 & 1.084 & 0.838 & 1.146 & 1.228 & 1.030 & 1.268 & 1.133 \\
\hline $\mathrm{Ti}$ & 0.319 & 0.391 & 0.224 & 0.212 & 0.241 & 0.207 & 0.254 & 0.328 & 0.219 & 0.210 & 0.222 & 0.329 \\
\hline $\mathrm{Fe}$ & 0.563 & 0.602 & 0.513 & 0.576 & 0.465 & 0.495 & 1.473 & 0.530 & 0.425 & 0.471 & 0.436 & 0.533 \\
\hline $\mathrm{Mn}$ & 0.000 & 0.004 & 0.006 & 0.008 & 0.004 & 0.006 & 0.026 & 0.002 & 0.005 & 0.007 & 0.006 & 0.000 \\
\hline $\mathrm{Mg}$ & 1.845 & 1.901 & 2.154 & 2.129 & 2.139 & 2.175 & 1.329 & 1.996 & 2.358 & 2.334 & 2.381 & 2.078 \\
\hline $\mathrm{Ca}$ & 0.032 & 0.000 & 0.000 & 0.000 & 0.000 & 0.005 & 0.012 & 0.004 & 0.009 & 0.005 & 0.007 & 0.003 \\
\hline $\mathrm{Na}$ & 0.021 & 0.010 & 0.010 & 0.013 & 0.016 & 0.013 & 0.000 & 0.032 & 0.023 & 0.038 & 0.026 & 0.040 \\
\hline $\mathrm{K}$ & 1.249 & 1.136 & 1.189 & 1.255 & 1.177 & 1.135 & 1.027 & 0.945 & 0.935 & 0.943 & 1.199 & 1.258 \\
\hline $\mathrm{Ba}$ & 0.005 & 0.009 & 0.042 & 0.017 & 0.068 & 0.035 & 0.006 & & & & 0.066 & 0.008 \\
\hline $\mathrm{Ni}$ & 0.004 & 0.005 & 0.000 & 0.004 & 0.000 & 0.061 & 0.000 & 0.003 & 0.000 & 0.000 & 0.002 & 0.004 \\
\hline $\mathrm{V}$ & 0.000 & 0.000 & 0.000 & 0.000 & 0.000 & 0.000 & 0.007 & 0.000 & 0.000 & 0.000 & 0.000 & 0.004 \\
\hline$S$ & & & & & & & & 0.006 & 0.000 & 0.000 & 0.000 & 0.007 \\
\hline $\mathrm{Cl}$ & & & & & & & & 0.004 & 0.000 & 0.000 & 0.000 & 0.006 \\
\hline Total & 7.945 & 7.889 & 8.046 & 8.066 & 8.030 & 8.021 & 7.901 & 7.797 & 7.925 & 7.901 & 8.184 & 8.082 \\
\hline
\end{tabular}

Note. P—phenocryst; G—groundmass; na—not analyzed; bdl—below detection limit. 
Table 3. Representative chemical compositions (wt.\%) and mineral formulae (apfu) of talk, saponite and garnet from the Vinoren aillikite.

\begin{tabular}{|c|c|c|c|c|c|c|}
\hline Analysis N & $4 a$ & $2-1 a$ & $4 c$ & $2 a$ & $2 b$ & $1 \mathrm{a}$ \\
\hline Mineral & Talk & Saponite & Saponite & Garnet & Garnet & Garnet \\
\hline $\mathrm{SiO}_{2}$ & 59.48 & 54.07 & 52.28 & 33.24 & 33.79 & 32.73 \\
\hline $\mathrm{TiO}_{2}$ & na & na & na & 0.30 & 1.16 & 0.99 \\
\hline $\mathrm{Al}_{2} \mathrm{O}_{3}$ & 0.04 & 3.51 & 2.24 & 1.36 & 1.94 & 0.81 \\
\hline $\mathrm{FeO}$ & 4.80 & 11.02 & 9.96 & na & na & na \\
\hline $\mathrm{Fe}_{2} \mathrm{O}_{3}$ & na & na & na & 29.36 & 27.31 & 29.08 \\
\hline $\mathrm{MnO}$ & 0.10 & 0.06 & 0.11 & 0.07 & 0.10 & 0.09 \\
\hline $\mathrm{MgO}$ & 24.86 & 24.07 & 21.88 & 0.40 & 0.93 & 0.05 \\
\hline $\mathrm{CaO}$ & 0.14 & 0.83 & 0.19 & 32.20 & 32.12 & 32.81 \\
\hline $\mathrm{Na}_{2} \mathrm{O}$ & bdl & 0.09 & 0.20 & na & na & na \\
\hline $\mathrm{K}_{2} \mathrm{O}$ & bdl & 0.14 & 0.34 & na & na & na \\
\hline $\mathrm{NiO}$ & bdl & 0.05 & 0.09 & na & na & na \\
\hline Total & 89.42 & 93.83 & 87.29 & 96.93 & 97.27 & 96.42 \\
\hline Formulae based on: & $11 \mathrm{O}$ & $11 \mathrm{O}$ & $11 \mathrm{O}$ & $12 \mathrm{O}$ & $12 \mathrm{O}$ & $12 \mathrm{O}$ \\
\hline $\mathrm{Si}$ & 4.081 & 3.703 & 3.828 & 2.898 & 2.907 & 2.877 \\
\hline $\mathrm{Ti}$ & & & & 0.020 & 0.075 & 0.065 \\
\hline $\mathrm{Al}$ & 0.003 & 0.283 & 0.193 & 0.140 & 0.197 & 0.084 \\
\hline $\mathrm{Fe}^{2+}$ & 0.275 & 0.631 & 0.610 & & & \\
\hline $\mathrm{Fe}^{3+}$ & & & & 1.926 & 1.768 & 1.924 \\
\hline $\mathrm{Mn}$ & 0.006 & 0.003 & 0.007 & 0.005 & 0.007 & 0.007 \\
\hline $\mathrm{Mg}$ & 2.542 & 2.458 & 2.388 & 0.052 & 0.119 & 0.007 \\
\hline $\mathrm{Ca}$ & 0.010 & 0.061 & 0.015 & 3.008 & 2.961 & 3.090 \\
\hline $\mathrm{Na}$ & 0.000 & 0.012 & 0.028 & & & \\
\hline K & 0.000 & 0.012 & 0.032 & & & \\
\hline $\mathrm{Ni}$ & 0.000 & 0.003 & 0.005 & & & \\
\hline Total & 6.918 & 7.167 & 7.106 & 8.049 & 8.035 & 8.054 \\
\hline
\end{tabular}

Note. na-not analyzed; bdl-below detection limit.

Table 4. Representative chemical compositions (wt.\%) and mineral formulae (apfu) of oxyspinel group minerals from the Vinoren aillikite.

\begin{tabular}{|c|c|c|c|c|c|c|c|c|c|c|c|c|c|c|}
\hline Analysis N & $2 d$ & $3-1 a$ & $4-1 a$ & $4-1 b$ & $7-1 a$ & $7-1 b$ & $8-1 a$ & $8-1 b$ & $9-1-3 a$ & $9-1-3 b$ & $4-1 a$ & $4-1 b$ & $5-1 c$ & $5-1 d$ \\
\hline & core & host & core & host & core & host & core & host & core & host & core & host & core & host \\
\hline $\mathrm{SiO}_{2}$ & 0.06 & 0.17 & 0.15 & 0.17 & 0.28 & 1.13 & 0.13 & 0.15 & 0.13 & 0.53 & 0.28 & 0.88 & 0.17 & 0.24 \\
\hline $\mathrm{Al}_{2} \mathrm{O}_{3}$ & 6.16 & 5.12 & 8.73 & 5.23 & 9.15 & 5.18 & 8.56 & 5.50 & 7.46 & 5.04 & 8.71 & 5.37 & 8.88 & 5.39 \\
\hline $\mathrm{TiO}_{2}$ & 6.42 & 10.48 & 6.91 & 8.88 & 7.79 & 9.38 & 7.61 & 9.84 & 6.46 & 7.46 & 8.31 & 10.74 & 8.58 & 10.24 \\
\hline $\mathrm{Cr}_{2} \mathrm{O}_{3}$ & 24.32 & 14.62 & 26.86 & 15.71 & 28.59 & 14.32 & 28.53 & 16.02 & 28.08 & 16.47 & 28.44 & 15.42 & 28.02 & 16.65 \\
\hline $\mathrm{Fe}_{2} \mathrm{O}_{3}$ & 49.84 & 63.63 & 43.99 & 64.95 & 39.80 & 59.69 & 42.88 & 60.38 & 44.32 & 65.45 & 43.60 & 61.47 & 43.30 & 60.21 \\
\hline $\mathrm{MnO}$ & 0.74 & 4.44 & 0.52 & 3.59 & 0.46 & 3.77 & 0.72 & 4.24 & 0.57 & 2.92 & 0.61 & 4.75 & 0.56 & 4.36 \\
\hline $\mathrm{MgO}$ & 7.00 & 0.13 & 8.72 & 0.23 & 9.24 & 0.81 & 8.69 & 0.15 & 7.93 & 0.70 & 8.67 & 0.18 & 8.72 & 0.08 \\
\hline $\mathrm{ZnO}$ & 0.21 & 1.64 & 0.22 & 1.06 & 0.15 & 1.46 & 0.24 & 1.57 & 0.14 & 0.83 & 0.24 & 1.51 & 0.17 & 1.68 \\
\hline $\mathrm{CaO}$ & 0.41 & 0.38 & 0.31 & 0.32 & 0.32 & 0.52 & 0.28 & 0.29 & 0.25 & 0.42 & 0.43 & 0.76 & 0.21 & 0.31 \\
\hline $\mathrm{NiO}$ & 0.15 & 0.19 & 0.25 & 0.24 & 0.22 & 0.11 & 0.22 & 0.11 & 0.11 & 0.24 & 0.32 & 0.19 & 0.24 & 0.19 \\
\hline $\mathrm{V}_{2} \mathrm{O}_{5}$ & na & 0.18 & 0.19 & 0.15 & 0.32 & 0.22 & 0.28 & 0.19 & 0.22 & 0.24 & na & na & na & na \\
\hline Total & 95.31 & 100.98 & 96.85 & 100.53 & 96.32 & 96.59 & 98.14 & 98.44 & 95.67 & 100.30 & 99.61 & 101.27 & 98.85 & 99.35 \\
\hline \multicolumn{15}{|c|}{ Mineral formulae on basis of 3 cations } \\
\hline $\mathrm{Si}$ & 0.002 & 0.006 & 0.005 & 0.006 & 0.010 & 0.044 & 0.005 & 0.006 & 0.005 & 0.020 & 0.010 & 0.033 & 0.006 & 0.009 \\
\hline $\mathrm{Al}$ & 0.270 & 0.228 & 0.366 & 0.233 & 0.382 & 0.238 & 0.355 & 0.250 & 0.320 & 0.224 & 0.355 & 0.237 & 0.365 & 0.243 \\
\hline $\mathrm{Ti}$ & 0.179 & 0.297 & 0.185 & 0.253 & 0.208 & 0.275 & 0.201 & 0.286 & 0.177 & 0.212 & 0.216 & 0.302 & 0.225 & 0.295 \\
\hline $\mathrm{Cr}$ & 0.714 & 0.436 & 0.755 & 0.470 & 0.801 & 0.442 & 0.793 & 0.489 & 0.809 & 0.491 & 0.778 & 0.456 & 0.772 & 0.503 \\
\hline $\mathrm{Fe}^{3+}$ & 1.393 & 1.806 & 1.176 & 1.849 & 1.061 & 1.753 & 1.134 & 1.754 & 1.215 & 1.859 & 1.135 & 1.729 & 1.136 & 1.732 \\
\hline $\mathrm{Mn}$ & 0.023 & 0.142 & 0.016 & 0.115 & 0.014 & 0.125 & 0.021 & 0.139 & 0.018 & 0.093 & 0.018 & 0.150 & 0.017 & 0.141 \\
\hline $\mathrm{Mg}$ & 0.388 & 0.007 & 0.462 & 0.013 & 0.488 & 0.047 & 0.455 & 0.009 & 0.431 & 0.039 & 0.447 & 0.010 & 0.453 & 0.005 \\
\hline $\mathrm{Zn}$ & 0.006 & 0.046 & 0.006 & 0.030 & 0.004 & 0.042 & 0.006 & 0.045 & 0.004 & 0.023 & 0.006 & 0.042 & 0.004 & 0.047 \\
\hline $\mathrm{Ca}$ & 0.016 & 0.015 & 0.012 & 0.013 & 0.012 & 0.022 & 0.011 & 0.012 & 0.010 & 0.017 & 0.016 & 0.030 & 0.008 & 0.013 \\
\hline $\mathrm{Ni}$ & 0.009 & 0.012 & 0.014 & 0.015 & 0.013 & 0.007 & 0.012 & 0.007 & 0.006 & 0.015 & 0.018 & 0.011 & 0.013 & 0.012 \\
\hline V & 0.000 & 0.004 & 0.004 & 0.004 & 0.007 & 0.006 & 0.007 & 0.005 & 0.005 & 0.006 & 0.000 & 0.000 & 0.000 & 0.000 \\
\hline$\sum$ cations & 3.000 & 3.000 & 3.000 & 3.000 & 3.000 & 3.000 & 3.000 & 3.000 & 3.000 & 3.000 & 3.000 & 3.000 & 3.000 & 3.000 \\
\hline
\end{tabular}


Table 5. Representative chemical compositions (wt.\%) and mineral formulae (apfu) of titanite, ilmenite, rutile and lukasite-(Ce) from the Vinoren aillikite.

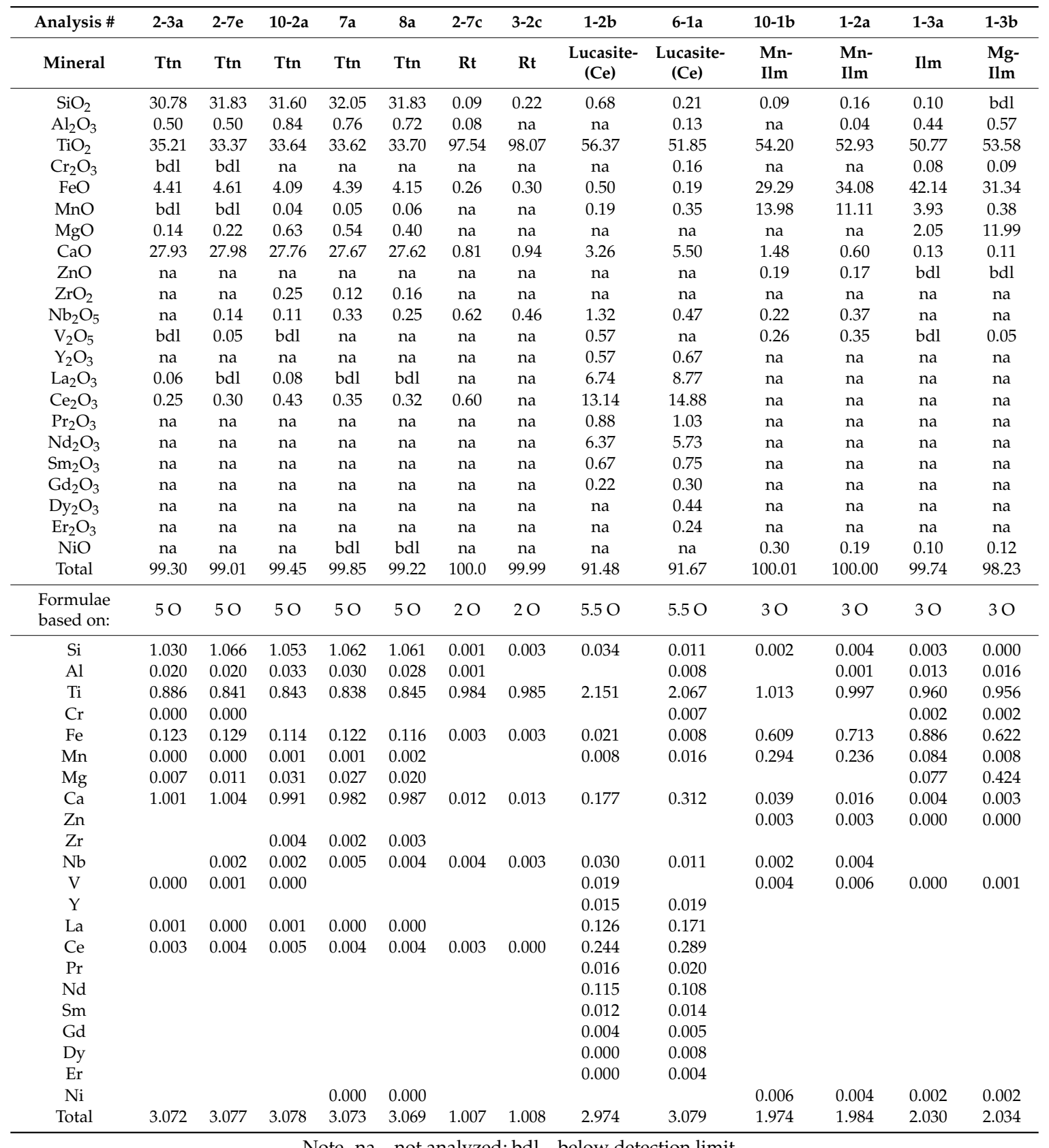

Note. na-not analyzed; bdl-below detection limit.

\subsection{Whole Rock Compositions}

The studied dyke rock is characterized by low $\mathrm{SiO}_{2}$ (34-35 wt.\%) and $\mathrm{Al}_{2} \mathrm{O}_{3}$ (5.1 wt.\%), moderate $\mathrm{TiO}_{2}$ (2.75 wt.\% in average) and $\mathrm{Mg} \#(75)$, high $\mathrm{CO}_{2}, \mathrm{P}_{2} \mathrm{O}_{5}, \mathrm{Ba}$ and $\mathrm{Sr}(9.8 \mathrm{wt} . \%, 1.1 \mathrm{wt} . \%, 2500 \mathrm{ppm}$ and $590 \mathrm{ppm}$ in average, respectively) (Table 6). From its composition and its ultrapotassic character $(\mathrm{K} / \mathrm{Na}=15$ in average), the Vinoren rock can be recognized as transitional between carbonatite and lamproite. The rock is different from lamproites since it is not peralkaline $\left(K_{a g p}<1\right)$ nor perpotassic $(\mathrm{K} / \mathrm{Al}<0.7)$. Furthermore, carbonatites and silicocarbonatites contain at least two times higher $\mathrm{CO}_{2}$ and significantly higher $\mathrm{Sr}$ than the rock from Vinoren [2,7]. The studied rock has high $\mathrm{Ni}$ (530-550 ppm) and Cr (630-750 ppm), typical for ultramafic volatile-rich mantle-derived magmas (average concentrations from [1]: UML $=430 \mathrm{ppm} \mathrm{Ni}, 480 \mathrm{ppm} \mathrm{Cr}$; kimberlites $=1050 \mathrm{ppm} \mathrm{Ni}$, 
$1100 \mathrm{ppm} \mathrm{Cr}$; lamproites $=435 \mathrm{ppm} \mathrm{Ni}, 510 \mathrm{ppm} \mathrm{Cr}$ ). Important to notice is the high content of volatile components of the rock, such as $\mathrm{F}(0.25-0.28 \mathrm{wt} . \%), \mathrm{S}(0.71-0.75 \mathrm{wt} . \%), \mathrm{H}_{2} \mathrm{O}(2.8-3.6 \mathrm{wt} . \%)$, and rare alkali elements (68 ppm Rb, 10 ppm Cs).

In the compositional variation diagram $\mathrm{MgO}-\mathrm{Al}_{2} \mathrm{O}_{3}-\mathrm{FeO}_{\text {tot }}$, the Vinoren rock plots well within the fields of kimberlite, melilitite, aillikite and alnöite, the latter two are UML (Figure 7). Compared to $\mathrm{UML}$, kimberlites have higher $\mathrm{MgO} / \mathrm{CaO}$ ratios, while melilitites have higher $\mathrm{Al}_{2} \mathrm{O}_{3} / \mathrm{CaO}$ ratios [38].

Table 6. Representative major and minor element analyses of the Vinoren aillikite.

\begin{tabular}{cccccc}
\hline Sample $\mathbf{N}$ & KK-1 & KK-3 & & KK-1 & KK-3 \\
\hline wt. \% & & & ppm & & \\
\hline $\mathrm{SiO}_{2}$ & 34.01 & 34.63 & $\mathrm{Ba}$ & 2780 & 2060 \\
$\mathrm{TiO}_{2}$ & 2.75 & 2.75 & $\mathrm{Sr}$ & 680 & 510 \\
$\mathrm{Al}_{2} \mathrm{O}_{3}$ & 5.11 & 5.05 & $\mathrm{Cu}$ & 91 & 100 \\
$\mathrm{Fe}_{2} \mathrm{O}_{3}$ & 3.44 & 2.93 & $\mathrm{Ni}$ & 530 & 550 \\
$\mathrm{FeO}$ & 4.37 & 4.73 & $\mathrm{Co}$ & 90 & 80 \\
$\mathrm{MnO}$ & 0.14 & 0.15 & $\mathrm{Cr}$ & 630 & 750 \\
$\mathrm{MgO}$ & 10.16 & 10.08 & $\mathrm{~V}$ & 110 & 170 \\
$\mathrm{CaO}$ & 19.36 & 19.41 & $\mathrm{Li}$ & 30 & 30 \\
$\mathrm{Na}_{2} \mathrm{O}$ & 0.09 & 0.12 & $\mathrm{Rb}$ & 68 & 68 \\
$\mathrm{~K}_{2} \mathrm{O}$ & 2.34 & 2.44 & $\mathrm{Cs}$ & 10 & 9 \\
$\mathrm{H}_{2} \mathrm{O}^{-}$ & 1.06 & 0.98 & & & \\
$\mathrm{LOI}$ & 3.6 & 2.79 & & & \\
$\mathrm{P}_{2} \mathrm{O}_{5}$ & 1.14 & 1.06 & & & \\
$\mathrm{~F}$ & 0.28 & 0.25 & & & \\
$\mathrm{~S}$ & 0.71 & 0.75 & & & \\
$\mathrm{CO}$ & 9.82 & 9.8 & & & \\
\hline $\mathrm{Mg} \#$ & 75 & 75 & & & \\
$\mathrm{~K}_{\mathrm{agp}}$ & 0.52 & 0.56 & & & \\
$\mathrm{~K} / \mathrm{Na}$ & 17 & 13 & & \\
$\mathrm{~K} / \mathrm{Al}$ & 0.50 & 0.52 & & & \\
\hline
\end{tabular}

Note. LOI-lost on ignition; Mg\# (magnesium number) $=\mathrm{Mg} /(\mathrm{Mg}+\mathrm{Fe})$.

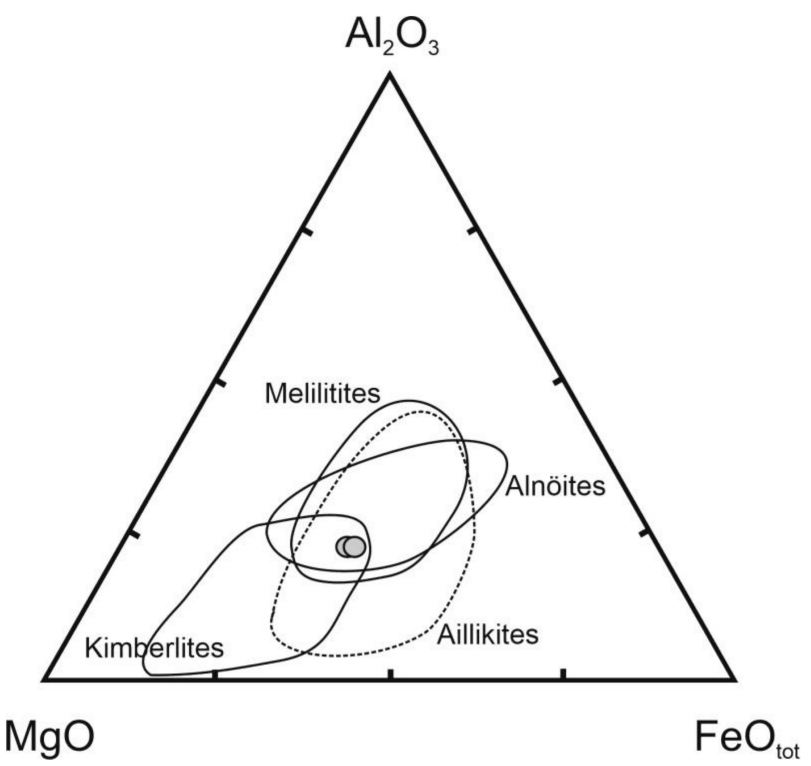

Figure 7. Whole rock compositional field for ultramafic lamprophyre, kimberlite and melilitite rocks (after [38]). Gray circles show data from this study for the Vinoren occurrence. 


\section{3. ${ }^{40} \mathrm{Ar} /{ }^{\beta 9} \mathrm{Ar}$ Geochronology}

Results and measurement conditions of ${ }^{40} \mathrm{Ar} /{ }^{39} \mathrm{Ar}$ analyses of Vinoren phlogopite are given in Table 7. Plateau was not obtained. But an arithmetic average age of $686 \pm 9 \mathrm{Ma}$ was calculated from the last 5 steps which show very similar ages (Figure 8a). The integrated ${ }^{40} \mathrm{Ar} /{ }^{39} \mathrm{Ar}$ age is $689 \pm 3 \mathrm{Ma}$. The measured $\mathrm{Ca} / \mathrm{K}$ ratios were very stable, indicating that phlogopite has not been affected by alteration or degassing processes. In the normal isotope correlation diagram in Figure $8 b$, the data yields an age of $679 \pm 6 \mathrm{Ma}$.
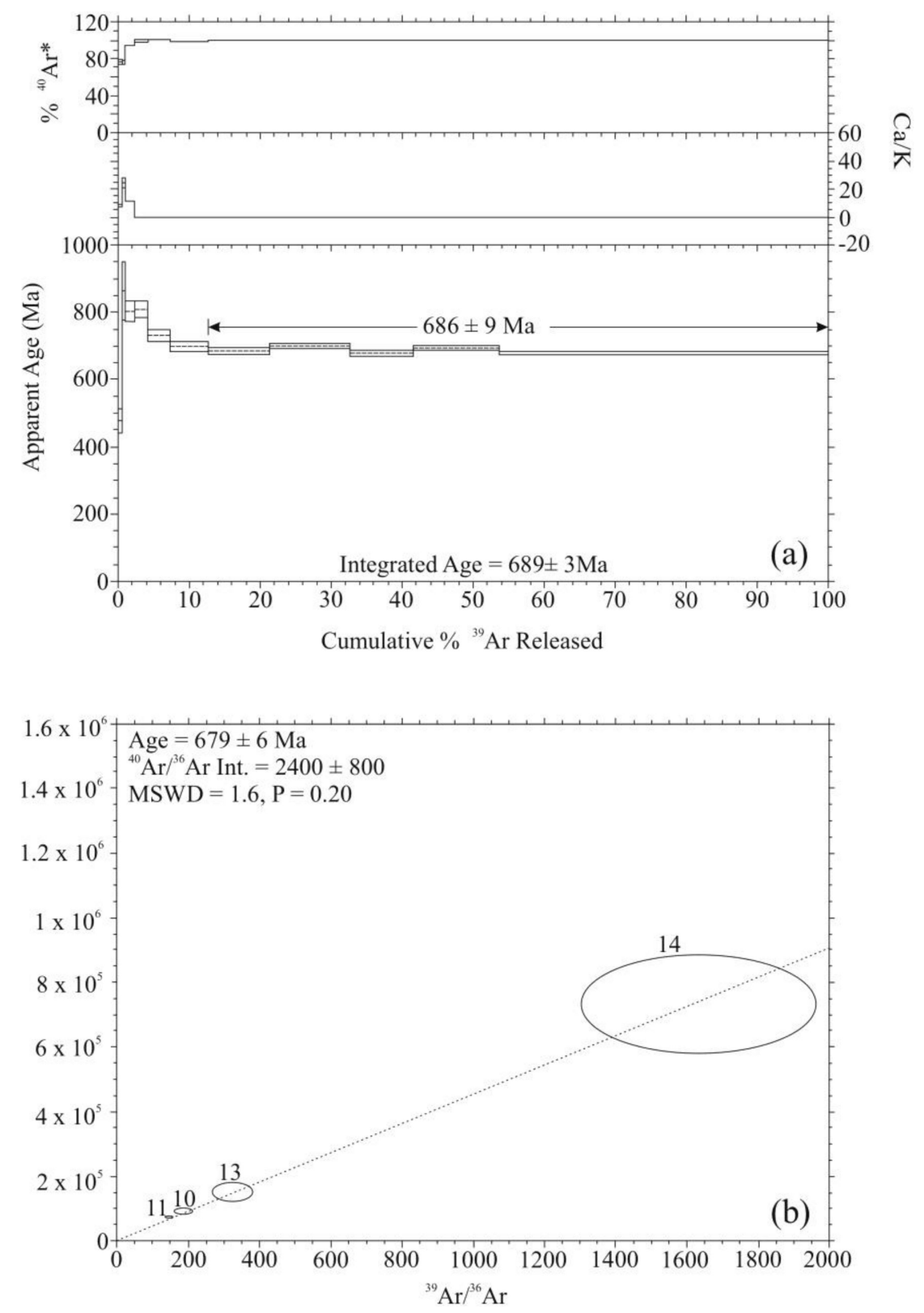

Figure 8. (a) Age spectrum for Vinoren phlogopite with an arithmetic average age of $686 \pm 9$ Ma of the last 5 steps, ${ }^{*}$ radiogenic ${ }^{40} \mathrm{Ar}$; (b) normal isochron for Vinoren phlogopite from the last five steps. 
Table 7. Results and measurements conditions of ${ }^{40} \mathrm{Ar} /{ }^{39} \mathrm{Ar}$ analyses of Vinoren phlogopite.

\begin{tabular}{|c|c|c|c|c|c|c|c|c|c|c|c|c|c|c|c|c|c|c|}
\hline \multirow{3}{*}{$\begin{array}{l}\text { Lab } \\
\text { ID\# }\end{array}$} & \multirow{3}{*}{$\begin{array}{c}\text { Laser } \\
\text { Power * } \\
(\%) \\
\end{array}$} & \multicolumn{10}{|c|}{ Relative Isotopic Abundances $\left(10^{-2} \mathrm{nA}\right)$} & \multirow{3}{*}{$\begin{array}{l}{ }^{39} \mathrm{Ar} \% \\
\text { of Total }\end{array}$} & & & & \multirow{3}{*}{\multicolumn{2}{|c|}{$\begin{array}{l}\text { Age (Ma) } \\
\quad \pm 1 \sigma\end{array}$}} & \multirow{3}{*}{$\begin{array}{l}w / \pm J \\
\pm 1 \sigma\end{array}$} \\
\hline & & \multirow{2}{*}{\multicolumn{2}{|c|}{$\begin{array}{l}{ }^{40} \mathrm{Ar} \\
\pm 1 \sigma\end{array}$}} & \multirow{2}{*}{\multicolumn{2}{|c|}{$\begin{array}{l}{ }^{39} \mathrm{Ar} \\
\pm 1 \sigma\end{array}$}} & \multirow{2}{*}{\multicolumn{2}{|c|}{$\begin{array}{l}{ }^{38} \mathrm{Ar} \\
\pm 1 \sigma\end{array}$}} & \multirow{2}{*}{\multicolumn{2}{|c|}{$\begin{array}{l}{ }^{37} \mathrm{Ar} \\
\pm \mathbf{1} \sigma\end{array}$}} & \multirow{2}{*}{\multicolumn{2}{|c|}{$\begin{array}{l}{ }^{36} \mathrm{Ar} \\
\pm 1 \sigma\end{array}$}} & & \multirow{2}{*}{\multicolumn{2}{|c|}{$\begin{array}{l}\mathrm{Ca} / \mathrm{K} \\
\pm 1 \sigma\end{array}$}} & & & & \\
\hline & & & & & & & & & & & & & & & & & & \\
\hline$E R-12-B t$ & & Jvaln & e: 1.004 & $10^{-3}$ & & Irrad & ation ID: & $\mathrm{O}-7$ & & & & & & & ${ }^{40} \mathrm{Ar}{ }^{* *}$ & & & \\
\hline 1098-01 & 1.4 & 9.2821 & 0.0999 & 0.009 & 0.0055 & 0.0061 & 0.0014 & 0.0271 & 0.0074 & 0.0251 & 0.0012 & 0.1 & 5.10 & 3.62 & 20.0 & 339.28 & 211.36 & 211.37 \\
\hline 1098-02 & 1.6 & 26.441 & 0.1962 & 0.0027 & 0.0021 & 0.0052 & 0.0013 & 0.0268 & 0.0078 & 0.0362 & 0.0012 & 0.0 & 17.13 & 15.43 & 59.6 & 3508.18 & 1324.35 & 1324.36 \\
\hline 1098-03 & 1.8 & 3.6442 & 0.0626 & 0.0002 & 0.0041 & 0.0008 & 0.0013 & 0.0009 & 0.0056 & 0.0174 & 0.0011 & 0.0 & 6.47 & 122.67 & 0.0 & & 0.00 & 0.00 \\
\hline 1098-04 & 2.0 & 37.526 & 0.2034 & 0.0945 & 0.0033 & 0.0137 & 0.0014 & 0.4424 & 0.0172 & 0.0317 & 0.0013 & 0.6 & 7.96 & 0.43 & 75.1 & 474.56 & 16.81 & 16.89 \\
\hline 1098-05 & 2.2 & 55.59 & 0.4316 & 0.0689 & 0.004 & 0.0166 & 0.0015 & 0.9673 & 0.016 & 0.0475 & 0.0013 & 0.4 & 23.87 & 1.52 & 74.9 & 862.55 & 43.74 & 43.83 \\
\hline 1098-06 & 2.5 & 141.37 & 0.3023 & 0.2383 & 0.0053 & 0.011 & 0.0015 & 1.4118 & 0.0193 & 0.0332 & 0.0012 & 1.4 & 10.07 & 0.28 & 93.2 & 799.31 & 15.50 & 15.71 \\
\hline 1098-07 & 2.7 & 190.26 & 0.4543 & 0.3344 & 0.0059 & 0.0062 & 0.0021 & 0.0456 & 0.0066 & 0.0081 & 0.0011 & 2.0 & 0.23 & 0.03 & 98.7 & 807.55 & 12.42 & 12.69 \\
\hline $1098-08$ & 2.9 & 250.51 & 0.633 & 0.5037 & 0.0073 & 0.0006 & 0.0019 & 0.0186 & 0.0062 & 0.0063 & 0.0011 & 3.0 & 0.06 & 0.02 & 99.3 & 726.82 & 9.43 & 9.73 \\
\hline 1098-09 & 3.1 & 435.14 & 0.6032 & 0.9182 & 0.0095 & 0.0131 & 0.0021 & 0.0094 & 0.0063 & 0.0187 & 0.0011 & 5.4 & 0.02 & 0.01 & 98.7 & 695.37 & 6.43 & 6.83 \\
\hline 1098-10 & 3.3 & 690.67 & 1.3019 & 1.5017 & 0.0111 & 0.0277 & 0.0018 & 0.0187 & 0.0057 & 0.008 & 0.001 & 8.8 & 0.02 & 0.01 & 99.7 & 683.52 & 4.67 & 5.19 \\
\hline 1098-11 & 3.5 & 904.32 & 2.0012 & 1.92 & 0.0121 & 0.0316 & 0.0023 & 0.0144 & 0.0072 & 0.0128 & 0.0012 & 11.2 & 0.01 & 0.01 & 99.6 & 696.72 & 4.16 & 4.76 \\
\hline 1098-12 & 3.7 & 694.88 & 1.202 & 1.5324 & 0.0111 & 0.0205 & 0.0022 & 0.0166 & 0.0079 & 0 & 0.001 & 9.0 & 0.02 & 0.01 & 100.1 & 677.90 & 4.52 & 5.06 \\
\hline $1098-13$ & 4.0 & 956.08 & 1.6007 & 2.0488 & 0.0131 & 0.0294 & 0.002 & 0.0768 & 0.0081 & 0.0063 & 0.0011 & 12.0 & 0.06 & 0.01 & 99.8 & 692.69 & 4.10 & 4.70 \\
\hline $1098-14$ & 6.0 & 3581.6 & 5.1002 & 7.9018 & 0.0271 & 0.1119 & 0.0034 & 0.4513 & 0.0125 & 0.0049 & 0.0011 & 46.3 & 0.10 & 0.00 & 100.0 & 676.99 & 2.30 & 3.22 \\
\hline
\end{tabular}

${ }^{*} 100 \%$ output of $\mathrm{CO}_{2}$ continuous laser corresponds to $50 \mathrm{~W}$; ** radiogenic ${ }^{40} \mathrm{Ar}$. 


\section{Discussion}

\subsection{Geochemical Constrains for Rock Affinity}

From its diagnostic mineralogy (carbonate-rich, but nepheline- and/or alkali feldspar- and melilite-absent; see Section 4.1) and whole rock geochemistry (low $\mathrm{SiO}_{2}$ and $\mathrm{Al}_{2} \mathrm{O}_{3}$, high $\mathrm{TiO}_{2}, \mathrm{CO}_{2}$, $\mathrm{P}_{2} \mathrm{O}_{5}, \mathrm{Ba}$ and Sr; see Section 4.2), the rock is classified as aillikite. According to [2], aillikite is a carbonate-rich member of the UML group derived from a volatile-rich, potassic, $\mathrm{SiO}_{2}$-poor magma.

The affinity and a possible source of the studied rock can be constrained by comparative studies. The nearest UML occurrences of similar age and tectonic setting are from the Labrador-Greenland areas, which are the parts of NAC. Two aillikite occurrences in these areas, i.e., Aillik Bay and Torngat, were chosen for comparison as their parental magmas originated at different depths [5,35,39]. The Aillik Bay aillikites are diamond-free, whereas the Torngat rocks are diamond-bearing with accessory mineral and xenocryst assemblages indicating a deep source. The Vinoren rock shows similar contents of $\mathrm{SiO}_{2}, \mathrm{Al}_{2} \mathrm{O}_{3}, \mathrm{~K}_{2} \mathrm{O}, \mathrm{CO}_{2}$ and $\mathrm{P}_{2} \mathrm{O}_{5}$ as the Torngat aillikite, but lower $\mathrm{MgO}, \mathrm{Na} 2 \mathrm{O}$ and higher $\mathrm{CaO}$ (Figure 9). At the same time the studied aillikite is differing from the Aillik Bay rocks by most components. It has been proposed that the Torngat ailikite was related to partial melting of metasomatized mantle (assemblages similar to MARID = mica-amphibole-rutile-ilmenite-diopside xenoliths from kimberlites [40]) during $\mathrm{CO}_{2}$ fluxing [7]. MARID nodules and veins are highly enriched in volatiles and incompatible elements [41,42] and according to [43], they crystallize within the diamond stability field, i.e., >4 GPa. Although aillikites are rich in $\mathrm{MgO}$ and Ni, their low $\mathrm{SiO}_{2}$ content and high contents of alkalis and volatiles suggest that they cannot be produced by melting of pure mantle peridotite. Foley [44] suggested a vein-plus-wall-rock melting mechanism for the generation of lamproitic magma. Accordingly, potassic and hydrous lamproitic magma can be produced by remelting of phlogopite-richterite-clinopyroxene dominated veins accommodated in peridotite of subcontinental lithospheric mantle (SCLM). Later, Foley et al. [45] and Tappe et al. [39] developed a similar model for the generation of UML melts, using a phlogopite-carbonate vein assemblage with minor apatite and Ti-oxide. Their remelting can produce potassic, hybrid carbonate-ultramafic silicate magma batches corresponding to aillikite melts. This has not been directly demonstrated yet, but the process is confirmed by experimental data [43], and encouraged by proximity of diamond-bearing aillikite and model MARID (see Figure 9). Both phlogopite and K-richterite can be present in MARID assemblages. However, the extremely high $\mathrm{K} / \mathrm{Na}$ of the Vinoren aillikite combined with its strongly Si-undersaturated character indicate a dominating role of phlogopite in the source, because melting of a richterite-dominated source would have given more Si-rich melts. The difference in $\mathrm{Na}$ and $\mathrm{K}$ composition between the natural products and model MARID-like material (Figure 9) can be explained by the extremely different proportions of amphibole and mica in MARID. The low $\mathrm{MgO} / \mathrm{CaO}$ ratio $(<1)$ of aillikite suggests that calcite is the dominating carbonate in the source. The high $\mathrm{TiO} 2$ content of aillikite (2.75 wt.\%) cannot be explained by melting of Ti-rich phlogopite only, suggesting the presence of ilmenite and/or rutile in the source [46]. 

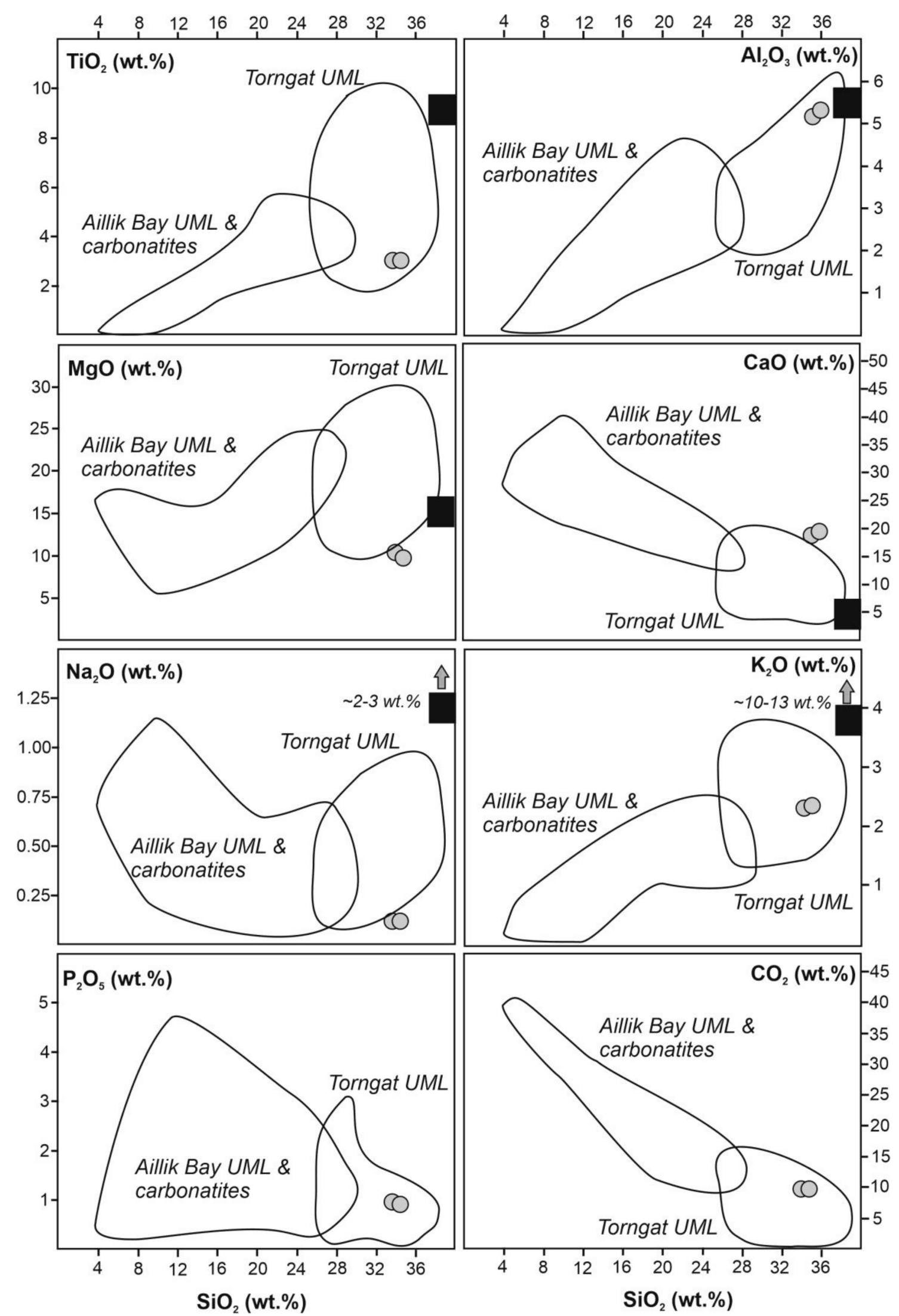

Figure 9. Major element oxide vs. $\mathrm{SiO}_{2}$ (wt.\%) of the Vinoren aillikite (gray circles). Also shown are the compositional fields of the diamond-bearing Torngat aillikite [35] and the diamond-free Aillik Bay aillikite [39] in Labrador which are of similar ages as the Vinoren rock. The black box shows the experimental melt compositions produced from MARID-type material [43]. 


\subsection{Mineralogical Constrains for Rock Genesis}

Minerals belonging to the phlogopite, oxyspinel and ilmenite groups may give important information about the mechanisms responsible for the genesis of volatile rich ultramafic rocks.

The chemical zonation observed for the groundmass phlogopite shows high kinoshitalite and tetraferriphlogopite components along the rim of the mineral. Kinoshitalite-rich rims are characteristic of kimberlitic mica [47], while tetraferriphlogopite rims are typical of lamproitic mica [36]. The elevated $\mathrm{BaO}$ content in phlogopite from Vinoren (up to $2.3 \mathrm{wt} . \%$ ) is much lower than what is observed from kimberlites, but higher than what is typical for phlogopite from aillikites. $\mathrm{BaO}$ content of $3.5 \mathrm{wt} . \%$ has been recognized in UML, including diamondiferous ones, from Australia [48,49]. The high $\mathrm{TiO}_{2}$ (4-7 wt.\%) in phlogopite from Vinoren is distinctly different from phlogopite from kimberlites and orangeites, but close to the compositions of phlogopite from UML and lamproites (Figure 10). Furthermore, the $\mathrm{Al}_{2} \mathrm{O}_{3}$ content in Vinoren phlogopite is different from high-Al kimberlitic phlogopite and low-Al orangeitic and lamproitic phlogopite. Phlogopite from orangeites and lamproites typically shows an evolutionary trend with an increase in Fe coupled with a decrease in Al toward pure tetraferriphlogopite. For phlogopite from the Vinoren rock, this trend is very weakly developed. In conclusion, phlogopite from Vinoren shows a hybrid character with some similarities to phlogopite from kimberlites and lamproites, but it is more similar to UML phlogopite, and it shows some affinity to MARID-like phlogopite (Figure 10).
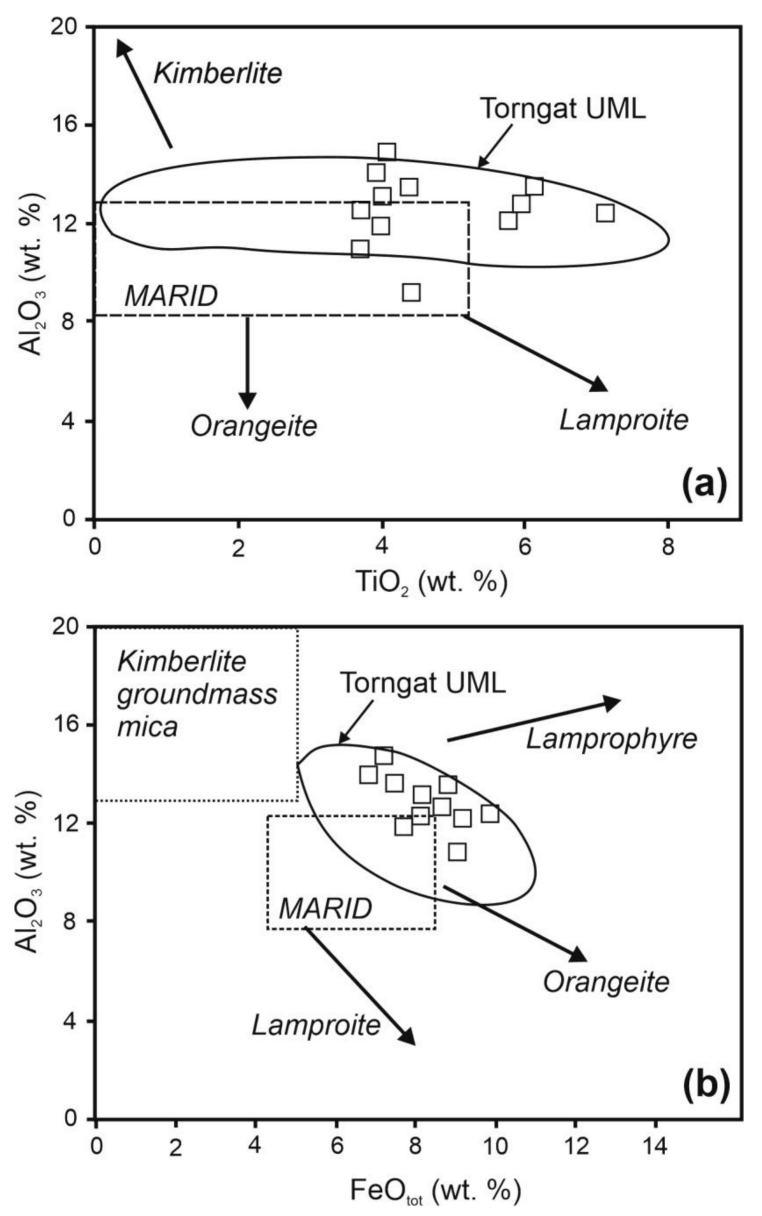

Figure 10. Compositional variations of phlogopite from the Vinoren rock in the diagrams (a) $\mathrm{TiO}_{2}$ vs. $\mathrm{Al}_{2} \mathrm{O}_{3}$ and (b) $\mathrm{FeO}_{\text {tot }}$ vs. $\mathrm{Al}_{2} \mathrm{O}_{3}$ (squares). Compositional fields and evolutionary trends of phlogopite from kimberlites, orangeites, lamproites and lamprophyres are after [50]. MARID (mica-amphibole-rutile-ilmenite-diopside) compositional field is after [40] and [51]. Phlogopite compositions from Torngat ultramafic lamprophyres (UML) are from [35]. 
The compositional variations of ilmenite from Vinoren indicate a hybrid nature also of this mineral (Figure 11). The Mg-rich core (up to $12 \mathrm{wt. \% )}$ is typical for kimberlitic ilmenite, while the more marginal part of the mineral is similar ilmenite from UML. The elevated MnO content (up to 3.9 wt. \%) may be considered as a result of the reaction trend in kimberlitic ilmenite as shown in Figure 11 [47,52,53]. Moreover, similar Mn-rich ilmenites have been observed as inclusions in diamonds from Brazil [54,55].

The manganoilmenite, which occurs as inclusions in titanite from the Vinoren rock, contains 11-14 wt.\% MnO. Compositions in this range are typical of ilmenite from carbonatites ([53,56], and references therein). However, ilmenite from carbonatites is commonly characterized by high $\mathrm{Nb}_{2} \mathrm{O}_{5}$ (1.1 wt.\% in average $\left.[47,56]\right)$. The manganoilmenite from Vinoren is depleted in $\mathrm{Nb}_{2} \mathrm{O}_{5}$ (0.2-0.4 wt.\%), which is more typical for ilmenite from kimberlites (0.22 wt.\% in average [56]).

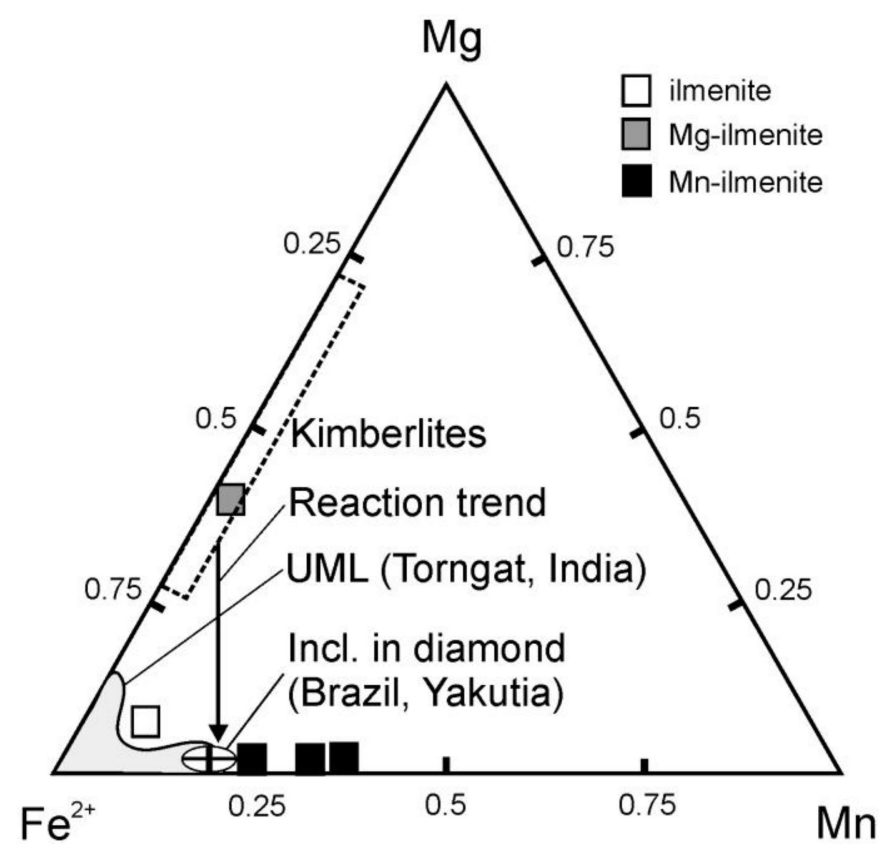

Figure 11. Composition of ilmenite group minerals from Vinoren compared to typical ilmenite from kimberlite [47], UML from Torngat [35], UML from India [57] and as inclusions in diamonds [54,55]. The reaction trend is after $[47,52]$.

Spinel from Vinoren commonly shows chemical zonation, reflecting changes in the chemical and physical conditions during mineral growth. Spinel shows $\mathrm{Fe}^{2+} /\left(\mathrm{Fe}^{2+}+\mathrm{Mg}\right)$ ratios in the range $0.3-0.5$ in cores and $0.85-1$ in the more marginal parts. Furthermore, there are the differences in $\mathrm{Ti} /(\mathrm{Ti}+\mathrm{Cr}+$ Al) (0.1-0.2 for cores and 0.2-0.4 for host grain), $\mathrm{Fe}^{3+} /\left(\mathrm{Fe}^{3+}+\mathrm{Cr}+\mathrm{Al}\right)(0.4-0.5$ and $0.5-0.8$, respectively), $\mathrm{Cr} /(\mathrm{Cr}+\mathrm{Al})\left(0.67-0.76\right.$ and $0.64-0.71$, respectively) and $\mathrm{Mn} /\left(\mathrm{Mn}+\mathrm{Fe}^{2+}\right)(0.05-0.06$ and $0.13-0.24$, respectively). In the diagrams $\mathrm{Fe}^{3+} /\left(\mathrm{Fe}^{3+}+\mathrm{Cr}+\mathrm{Al}\right)$ vs. $\mathrm{Fe}^{2+} /\left(\mathrm{Fe}^{2+}+\mathrm{Mg}\right)$ and $\mathrm{Fe}^{3+} /\left(\mathrm{Fe}^{3+}+\mathrm{Cr}+\mathrm{Al}\right)$ vs. $\mathrm{Fe}^{2+} /\left(\mathrm{Fe}^{2+}+\mathrm{Mg}\right)$, spinel cores show compositions corresponding to "magnesian ulvöspinel" and "Cr-spinel" from kimberlites (Figure 12a,b). The more marginal parts of spinel plot within the field of "titanomagnetite" from lamproites and UML. Thus, it can be inferred that the earliest spinel originated from deep "kimberlite-producing" levels, while the later spinel formed at shallower "UML" levels. The xenocrystic nature of Vinoren spinel (see Section 4.1) confirms this assumption. The marginal parts of spinel grains are usually enriched in Mn compared to the cores (Figure 12c), which is in accordance with an overall higher Mn activity at a late carbonatization stage of the Vinoren aillikite. The assemblage of magnesian ulvöspinel and Cr-spinel of kimberlitic affinity in association with Mg-ilmenite is widely recognized as an indicator for diamond ([47,58], and references therein).

Thus, phlogopite, ilmenite and spinel from the studied rock show compositions that suggest a hybrid and multistage origin of the rock. It is inferred that a primary melt originated from deep (kimberlitic) and possibly diamond-bearing mantle levels. Phlogopite compositions indicate that the 
melt originated from MARID-like source. During the ascend, the residual silicate melt with significant carbonate content was still reactive and resulted in the formation of ilmenite, manganilmenite and titanomagnetitic spinel at shallower (UML) mantle levels.
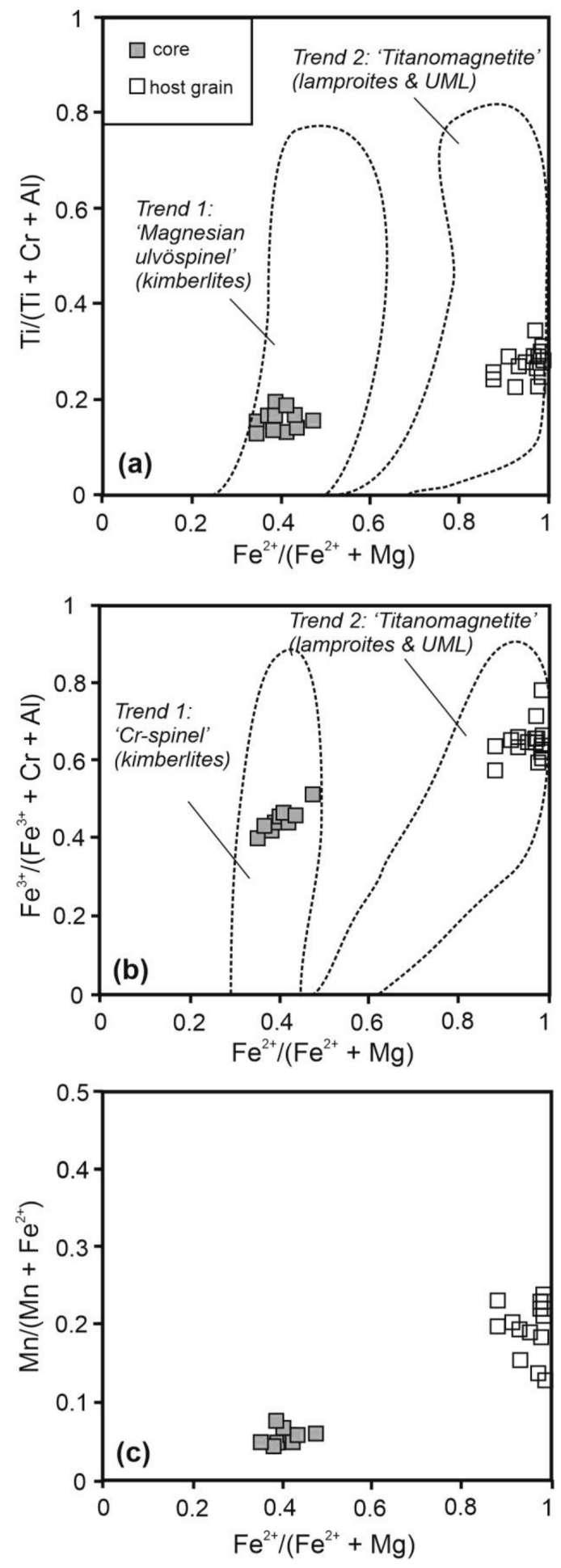

Figure 12. Variations in (a) $\mathrm{Ti} /(\mathrm{Ti}+\mathrm{Cr}+\mathrm{Al}),(\mathbf{b}) \mathrm{Fe}^{3+} /\left(\mathrm{Fe}^{3+}+\mathrm{Cr}+\mathrm{Al}\right)$ and (c) $\mathrm{Mn} /\left(\mathrm{Mn}+\mathrm{Fe}^{2+}\right)$ vs. $\mathrm{Fe}^{2+} /\left(\mathrm{Fe}^{2+}+\mathrm{Mg}\right)$ of spinel from the studied rock. The compositional fields for magnesian ulvöspinel/Cr-spinel from kimberlites (trend 1) and titanomagnetite from lamproites and UML (trend 2) are from $[39,50]$. 


\subsection{Possible Geodynamic Setting of Vinoren Aillikite}

The North Atlantic Craton of Rodinia is composed of Archean blocks surrounded by Paleoproterozoic mobile belts covering large areas in the Northeastern Quebec, Labrador and Western Greenland ([15], and references therein). Widespread lithospheric thinning occurred throughout eastern NAC along the Laurentian margin during the Late Neoproterozoic [59-62], resulting in continental breakup and subsequent opening of the Iapetus Ocean at $600 \mathrm{Ma}$, which was associated with rift-related UML-carbonatite-kimberlite magmatism. In central Labrador, this episode of continental stretching is recorded by remnant graben structures forming the eastward continuation of the St. Lawrence valley rift system [63]. Although Baltica today is separated from Laurentia, the two continents probably shared a common drift history during the time interval 750-600 Ma.

Studies of Neoproterozoic sedimentary systems along the northwestern region of Baltica, and geochemical and geochronological studies of magmatic rocks in the same region, have been used to constrain the break-up of Rodinia [60,64,65]. Prior to the active rift-related drift at ca. 600-550 Ma [66,67], this margin was inferred to have faced Laurentia (e.g., [68-70]).

During this stage, with thin SCLM and shallow asthenosphere, several carbonatitic-ultramafic complexes formed, including the Fen Complex in South Norway [71,72], the Seiland Igneous Province in North Norway (e.g., [73]) and the Alnö Carbonatite Complex in Sweden [74,75]. The initiation of rifting along the Baltic margin is marked by the $650 \mathrm{Ma}$ Egersund tholeiitic dykes (SW Norway) which probably were derived from a mantle plume [60]. The emplacement of the Vinoren aillikite pre-dates this event. This is in accordance with the concept of [76] suggesting that continental extension was going on from 750 to $530 \mathrm{Ma}$, but separated in two distinct phases: (1) At 750-680 Ma, and (2) at 615-550 Ma. The first phase marked a failed rifting event between Laurentia and Amazonia, while the second phase led to the final breakup of Rodinia and the opening of the Iapetus ocean. Our data show that the first phase was active also between Laurentia and Baltica. The geochemical and mineralogical data presented here suggest that the parental magma of the dyke originated under a relatively thick SCLM, and that the continental root might have reached the depth of diamond stability.

\section{Conclusions}

(1) From petrography and diagnostic mineralogy, the Vinoren rock can be classified as aillikite-carbonate-rich member of the UML group derived from a volatile-rich, potassic, $\mathrm{SiO}_{2}$-poor magma.

(2) The Vinoren aillikite has whole rock contents of $\mathrm{SiO}_{2}, \mathrm{Al}_{2} \mathrm{O}_{3}, \mathrm{~K}_{2} \mathrm{O}, \mathrm{CO}_{2}$ and $\mathrm{P}_{2} \mathrm{O}_{5}$ and phlogopite compositions similar to diamond-bearing aillikites (e.g., from Torngat, Labrador), having a MARID-like mantle source.

(3) The rock affinity and the age of the Vinoren aillikite indicate that the rock belongs to the spacious Neoproterozoic UML-kimberlite-carbonatite province of North Atlantic craton.

(4) Xenocrystic ilmenite and spinel have compositional characteristics of minerals forming in the diamond stability depth $(>130 \mathrm{~km})$.

(5) The emplacement of Vinoren aillikte pre-dates the rifting and breakup of Rodinia in North-Western Baltica and its parent magma formed under a thick SCLM.

Supplementary Materials: The following is available online at http://www.mdpi.com/2075-163X/10/11/1029/s1, Table S1: Chemical composition of oxyspinel group minerals from Vinoren aillikite.

Author Contributions: Conceptualization, D.R.Z. and K.K.; Methodology, Y.E.S. and M.S.; Investigation, D.R.Z., K.K., E.R., U.A. and M.S.; Writing-Original Draft Preparation, D.R.Z. and K.K.; Writing-Review and Editing, K.K., E.R., U.A.; Visualization, D.R.Z., K.K. and Y.E.S. All authors have read and agreed to the published version of the manuscript.

Funding: This research was funded by Russian Government grant 0226-2019-0053.

Acknowledgments: Thanks to Christian Berg for the photo in Figure 2a, and to Egil Olafsen for giving us access to the Ringnesgangen mine. Anna Solovjova (KSC RAS) assisted with drawing. Three anonymous referees are thanked for very helpful journal reviews. 
Conflicts of Interest: The authors declare no conflict of interest.

\section{References}

1. Rock, N.M.S. Lamprophyres; Blackie and Sons Ltd.: Glasgow, UK, 1991; p. 285.

2. Tappe, S.; Foley, S.F.; Jenner, G.A.; Kjarsgaard, B.A. Integrating Ultramafic Lamprophyres into the IUGS Classification of Igneous Rocks: Rationale and Implications. J. Pet. 2005, 46, 1893-1900. [CrossRef]

3. Hamilton, R. Geology and structural setting of ultramafic lamprophyres from Bulljah Pool, central Western Australia. J. R. Soc. West. Aust. 1992, 75, 51-56.

4. Mitchell, R.; Smith, B.S.; Larsen, L. Mineralogy of Ultramafic Dikes from The Sarfartoq, Sisimiut and Maniitsoq Areas, West Greenland: Kimberlites Or Melnoites? In Proceedings of the International Kimberlite Conference, Cape Town, South Africa, 11-17 April 1998.

5. Digonnet, S.; Goulet, N.; Bourne, J.; Stevenson, R.; Archibald, D. Petrology of the Abloviak Aillikite dykes, New Québec: Evidence for a Cambrian diamondiferous alkaline province in northeastern North America. Can. J. Earth Sci. 2000, 37, 517-533. [CrossRef]

6. Birkett, T.; McCandless, T.; Hood, C. Petrology of the Renard igneous bodies: Host rocks for diamond in the northern Otish Mountains region, Quebec. Lithos 2004, 76, 475-490. [CrossRef]

7. Tappe, S.; Foley, S.F.; Kjarsgaard, B.A.; Romer, R.L.; Heaman, L.M.; Stracke, A.; Jenner, G.A. Between carbonatite and lamproite-Diamondiferous Torngat ultramafic lamprophyres formed by carbonate-fluxed melting of cratonic MARID-type metasomes. Geochim. Cosmochim. Acta 2008, 72, 3258-3286. [CrossRef]

8. Hutchison, M.T.; Frei, D. Kimberlite and related rocks from Garnet Lake, West Greenland, including their mantle constituents, diamond occurrence, age and provenance. Lithos 2009, 112, 318-333. [CrossRef]

9. O'Brien, H.E.; Peltonen, P.; Vartianen, H. Kimberlites, Carbonatites, and Alkaline Rocks. In Precambrian Geology of Finland-Key to the Evolution of the Fennoscandian Shield. Developments in Precambrian Geology 14; Lehtinen, M., Nurmi, P.A., Ramo, O.T., Eds.; Elsevier B.V.: Amsterdam, The Netherlands, 2005; pp. 605-644.

10. Dalton, H.; Giuliani, A.; O'Brien, H.; Phillips, D.; Hergt, J. The role of lithospheric heterogeneity on the composition of kimberlite magmas from a single field: The case of Kaavi-Kuopio, Finland. Lithos 2020, 105333. [CrossRef]

11. Berthelsen, A. Towards a palinspastic tectonic analysis of the Baltic Shield. In Geology of Europe, from Precambrian to the post-Hercyninan Sedimentary Basins; Cogne, J., Slansky, M., Eds.; Mémoires du B.R.G.M.: Paris, France, 1980; pp. 5-21.

12. Demaiffe, D.; Michot, J. Isotope Geochronology of the Proterozoic Crustal Segment of Southern Norway: A Review. In The Deep Proterozoic Crust in the North Atlantic Provinces; Springer Science and Business Media LLC: Berlin, Germany, 1985; Volume C158, pp. 411-433.

13. Falkum, T. Geotectonic Evolution of Southern Scandinavia in Light of a Late-Proterozoic Plate-Collision. In The Deep Proterozoic Crust in the North Atlantic Provinces; Springer Science and Business Media LLC: Berlin, Germany, 1985; pp. 309-322.

14. Hoffman, P.F. Did the Breakout of Laurentia Turn Gondwanaland Inside-Out? Science 1991, 252, $1409-1412$. [CrossRef]

15. Li, Z.; Bogdanova, S.; Collins, A.; Davidson, A.; De Waele, B.; Ernst, R.; Fitzsimons, I.; Fuck, R.; Gladkochub, D.; Jacobs, J.; et al. Assembly, configuration, and break-up history of Rodinia: A synthesis. Precambrian Res. 2008, 160, 179-210. [CrossRef]

16. Bingen, B.; Davis, W.J.; Hamilton, M.A.; Engvik, A.K.; Stein, H.J.; Skar, O.; Nordgulen, O. Geochronology of high-grade metamorphism in the Sveconorwegian belt, S. Norway: U-Pb, Th-Pb and Re-Os data. Nor. J. Geol. 2008, 88, 13-42.

17. Bingen, B.; Viola, G. The early-Sveconorwegian orogeny in southern Norway: Tectonic model involving delamination of the sub-continental lithospheric mantle. Precambrian Res. 2018, 313, 170-204. [CrossRef]

18. Slagstad, T.; Roberts, N.M.W.; Marker, M.; Røhr, T.S.; Schiellerup, H. A non-collisional, accretionary Sveconorwegian orogen. Terra Nova 2012, 25, 30-37. [CrossRef]

19. Slagstad, T.; Roberts, N.M.W.; Coint, N.; Høy, I.; Sauer, S.; Kirkland, C.; Marker, M.; Røhr, T.S.; Henderson, I.H.; Stormoen, M.A.; et al. Magma-driven, high-grade metamorphism in the Sveconorwegian Province, southwest Norway, during the terminal stages of Fennoscandian Shield evolution. Geosphere 2018, 14, 861-882. [CrossRef] 
20. Bingen, B.; Nordgulen, Ø.; Viola, G. A four-phase model for the Sveconorwegian orogeny, SW Scandinavia. Nor. J. Geol. 2008, 88, 43-72.

21. Viola, G.; Bingen, B.; Solli, A. Berggrunnskart: Kongsberg litotektoniske enhet, Kongsberg-Modum-Hønefoss M 1: 100 000. Nor. Geol. Undersøkelse 2016. Available online: www.ngu.no/upload/Publikasjoner/Kart/ KongsbergB100.pdf (accessed on 17 November 2020).

22. Bugge, C. Kongsbergfeltets geologi. Nor. Geol. Undersøkelse 1917, 82, 272.

23. Gammon, J.B. Fahlbands in the Precambrian of southern Norway. Econ. Geol. 1966, 61, 174-188. [CrossRef]

24. Ineson, P.R.; Mitchell, J.G.; Vokes, F.M. K-Ar dating of epigenetic mineral deposits; an investigation of the Permian metallogenic province of the Oslo region, southern Norway. Econ. Geol. 1975, 70, 1426-1436. [CrossRef]

25. Ihlen, P.M.; Ineson, P.R.; Mitchell, J.G.; Vokes, F.M. K-Ar Dating of Dolerite Dykes in the Kongsberg-Fiskum District, Norway, and Their Relationships with the Silver and Base-Metal Veins. Nor. Geol. Undersøkelse 1984, 64, 87-96.

26. Torgersen, E.; Viola, G.; Zwingmann, H.; Henderson, I.H. Inclined K-Ar illite age spectra in brittle fault gouges: Effects of fault reactivation and wall-rock contamination. Terra Nova 2014, 27, 106-113. [CrossRef]

27. Bugge, A. Gammel og ny geologi ved Kongsberg Sølvverk. Nor. Geol. Undersøkelse 1932, 12, 74-89.

28. Neumann, H. Silver deposits at Kongsberg. Nor. Geol. Undersøkelse 1944, 162, 133.

29. Kotková, J.; Kullerud, K.; Šrein, V.; Drábek, M.; Škoda, R. The Kongsberg silver deposits, Norway: Ag-Hg-Sb mineralization and constraints for the formation of the deposits. Miner. Deposita 2017, 53, 531-545. [CrossRef]

30. Cazes, J. Ewing's Analytical Instrumentation Handbook, 3rd ed.; Marcel Dekker: New York, NY, USA, 2005; p. 1037.

31. Uto, K.; Ishizuka, O.; Matsumoto, A.; Kamioka, H.; Togashi, S. Laser-heating 40 Ar/39 Ar dating system of the Geological Survey of Japan: System outlines and preliminary results. Bull. Geol. Surv. Jpn. 1997, 48, $23-46$.

32. Ishizuka, O.; Yuasa, M.; Uto, K. Evidence of porphyry copper-type hydrothermal activity from a submerged remnant back-arc volcano of the Izu-Bonin arc. Earth Planet. Sci. Lett. 2002, 198, 381-399. [CrossRef]

33. Steiger, R.; Jäger, E. Subcommission on geochronology: Convention on the use of decay constants in geoand cosmochronology. Earth Planet. Sci. Lett. 1977, 36, 359-362. [CrossRef]

34. Chanturiya, V.A.; Minenko, V.G.; Makarov, D.V.; Suvorova, O.V.; Selivanova, E. Advanced Techniques of Saponite Recovery from Diamond Processing Plant Water and Areas of Saponite Application. Minerals 2018, 8, 549. [CrossRef]

35. Tappe, S.; Jenner, G.A.; Foley, S.; Heaman, L.; Besserer, D.; Kjarsgaard, B.A.; Ryan, B. Torngat ultramafic lamprophyres and their relation to the North Atlantic Alkaline Province. Lithos 2004, 76, 491-518. [CrossRef]

36. Mitchell, R.H.; Bergman, S.C. Petrology of Lamproites; Springer: Boston, MA, USA, 1991; ISBN 978-1-4613-6688-1.

37. Nickel, E.H.; Grey, I.E.; Madsen, I.C. Lucasite-(Ce), CeTi2(O,OH)6, a New Mineral from Western-Australia-Its Description and Structure. Am. Mineral. 1987, 72, 1006-1010.

38. Rock, N.M.S. The Nature and Origin of Ultramafic Lamprophyres: Aln ites and Allied Rocks. J. Pet. 1986, 27, 155-196. [CrossRef]

39. Tappe, S.; Foley, S.F.; Jenner, G.A.; Heaman, L.M.; Kjarsgaard, B.A.; Romer, R.L.; Stracke, A.; Joyce, N.; Hoefs, J. Genesis of Ultramafic Lamprophyres and Carbonatites at Aillik Bay, Labrador: A Consequence of Incipient Lithospheric Thinning beneath the North Atlantic Craton. J. Pet. 2006, 47, 1261-1315. [CrossRef]

40. Dawson, J.; Smith, J.V. The MARID (mica-amphibole-rutile-ilmenite-diopside) suite of xenoliths in kimberlite. Geochim. Cosmochim. Acta 1977, 41, 309-323. [CrossRef]

41. Kramers, J.; Roddick, J.; Dawson, J. Trace element and isotope studies on veined, metasomatic and "MARID" xenoliths from Bultfontein, South Africa. Earth Planet. Sci. Lett. 1983, 65, 90-106. [CrossRef]

42. Grégoire, M.; Bell, D.; Le Roex, A. Trace element geochemistry of phlogopite-rich mafic mantle xenoliths: Their classification and their relationship to phlogopite-bearing peridotites and kimberlites revisited. Contrib. Miner. Pet. 2002, 142, 603-625. [CrossRef]

43. Konzett, J. Phase relations and chemistry of Ti-rich K-richterite-bearing mantle assemblages: An experimental study to $8.0 \mathrm{GPa}$ in a Ti-KNCMASH system. Contrib. Miner. Pet. 1997, 128, 385-404. [CrossRef]

44. Foley, S. Vein-plus-wall-rock melting mechanisms in the lithosphere and the origin of potassic alkaline magmas. Lithos 1992, 28, 435-453. [CrossRef] 
45. Foley, S.; Andronikov, A.V.; Melzer, S. Petrology of ultramafic lamprophyres from the Beaver Lake area of Eastern Antarctica and their relation to the breakup of Gondwanaland. Miner. Pet. 2002, 74, 361-384. [CrossRef]

46. Foley, S.F.; Musselwhite, D.S.; van der Laan, S.R. Melt compositions from ultramafic vein assemblages in the lithospheric mantle: A comparison of cratonic and non-cratonic settings. In Proceedings of the 7th International Kimberlite Conference, Cape Town, South Africa, 11-17 April 1999; Gurney, J.J., Gurney, J.L., Pascoe, M.D., Richardson, S.H., Eds.; Red Roof Design: Cape Town, South Africa, 1999; pp. 238-246.

47. Mitchell, R.H. Kimberlites: Mineralogy, Geochemistry and Petrology; Plenum Press: New York, NY, USA, 1986; p. 442.

48. Hamilton, R.; Rock, N.M. Geochemistry, mineralogy and petrology of a new find of ultramafic lamprophyres from Bulljah Pool, Nabberu Basin, Yilgarn Craton, Western Australia. Lithos 1990, 24, 275-290. [CrossRef]

49. Sheppard, S.; Taylor, W.R. Barium- and LREE-rich, olivine-mica-lamprophyres with affinities to lamproites, Mt. Bundey, Northern Territory, Australia. Lithos 1992, 28, 303-325. [CrossRef]

50. Mitchell, R.H. Kimberlites, Orangeites and Related Rocks; Plenum Press: New York, NY, USA, 1995; p. 410.

51. Smith, J.; Brennesholtz, R.; Dawson, J. Chemistry of micas from kimberlites and xenoliths-I. Micaceous kimberlites. Geochim. Cosmochim. Acta 1978, 42, 959-971. [CrossRef]

52. Wyatt, G.R. Manganoan Ilmenite from the Premier kimberlite. In Kimberlite Symposium II Extended Abstract; American Geophysical Union: Washington, DC, USA, 1979; pp. 279-284.

53. Chakhmouradian, A.R.; Mitchell, R.H. Niobian ilmenite, hydroxylapatite and sulfatian monazite: Alternative hosts for incompatible elements in calcite kimberlite from Internatsional'naya, Yakutia. Can. Mineral. 1999, 37, 1177-1189.

54. Kaminsky, F.V.; Zakharchenko, O.; Davies, R.; Griffin, W.L.; Khachatryan-Blinova, G.; Shiryaev, A. Superdeep diamonds from the Juina area, Mato Grosso State, Brazil. Contrib. Miner. Pet. 2001, 140, 734-753. [CrossRef]

55. Kaminsky, F.; Belousova, E. Manganoan ilmenite as kimberlite/diamond indicator mineral. Russ. Geol. Geophys. 2009, 50, 1212-1220. [CrossRef]

56. Garanin, V.K.; Kudryavtseva, G.P.; Lapin, A.V. Typical features of ilmenite from kimberlites, alkali-ultrabasic intrusions, and carbonatites. Int. Geol. Rev. 1980, 22, 1025-1050. [CrossRef]

57. Nayak, B.; Meyer, F.M. Manganilmenite in the Magnetite Ore Body from Pokphur Area of Nagaland, North East India and the Possibility of Microdiamonds in the Ophiolites of Indo-Myanmar Ranges. Curr. Sci. 2017, 112, 155. [CrossRef]

58. Schulze, D.J. Diamonds-Theory and Exploration, A guide to the recognition and significance of kimberlite indicator minerals. Geol. Assoc. Can. Short Course 1995, 20, 1-39.

59. Bond, G.C.; Nickeson, P.A.; Kominz, M.A. Breakup of a supercontinent between $625 \mathrm{Ma}$ and $555 \mathrm{Ma}$ : New evidence and implications for continental histories. Earth Planet. Sci. Lett. 1984, 70, 325-345. [CrossRef]

60. Torsvik, T.; Smethurst, M.; Meert, J.; VanderVoo, R.; McKerrow, W.; Brasier, M.; Sturt, B.; Walderhaug, H. Continental break-up and collision in the Neoproterozoic and Palaeozoic-A tale of Baltica and Laurentia. Earth Sci. Rev. 1996, 40, 229-258. [CrossRef]

61. Cawood, P.A.; McCausland, P.J.A.; Dunning, G.R. Opening Iapetus: Constraints from the Laurentian margin in Newfoundland. Geol. Soc. Am. Bull. 2001, 113, 443-453. [CrossRef]

62. Puffer, J.H. A late Neoproterozoic eastern Laurentian superplume: Location, size, chemical composition, and environmental impact. Am. J. Sci. 2002, 302, 1-27. [CrossRef]

63. Gower, C.F.; Erdmer, P.; Wardle, R.J. The Double Mer Formation and the Lake Melville rift system, eastern Labrador. Can. J. Earth Sci. 1986, 23, 359-368. [CrossRef]

64. Kathol, B. Evolution of the rifted and subducted Late Proterozoic to Early Paleozoic Baltoscandian margin in the Torneträsk section, northern Swedish Caledonides. Stockh. Contrib. Geol. 1987, 42, 1-83.

65. Andréasson, P.-G.; Albrecht, L. Derivation of 500 Ma eclogites from the passive margin of Baltica and a note on the tectonometamorphic heterogeneity of eclogite-bearing crust. Geol. Mag. 1995, 132, 729-738. [CrossRef]

66. Siedlecka, A.; Roberts, D.; Nystuen, J.P.; Olovyanishikov, V.G. Northeastern and northwestern margin of Baltica in Neoproterozoic time: Evidence from the Timan and Caledonian orogens. In The Neoproterozoic Timanide Orogen of eastern Baltica; Gee, D.G., Pease, V.L., Eds.; Geological Society: London, UK, 2004; pp. 169-190. 
67. Pease, V.; Daly, J.; Elming, S.-Å.; Kumpulainen, R.; Moczydlowska, M.; Puchkov, V.; Roberts, D.; Saintot, A.; Stephenson, R. Baltica in the Cryogenian, 850-630Ma. Precambrian Res. 2008, 160, 46-65. [CrossRef]

68. Roberts, D.; Gale, G.H. The Caledonian-Appalachian Iapetus Ocean. In Evolution of the Earth's Crust; Tarling, D.H., Ed.; Academic Press: Cambridge, MA, USA, 1978; pp. 255-324.

69. Gower, C.F.; Ryan, A.B.; Rivers, T. Mid-Proterozoic Laurentia-Baltica: An overview of its geological evolution and summary of the contributions by this volume. Geol. Assoc. Canada Spec. Pap. 1990, 38, 1-20.

70. Gorbatschev, R.; Bogdanova, S. Frontiers in the Baltic Shield. Precambrian Res. 1993, 64, 3-21. [CrossRef]

71. Griffin, W.L.; Taylor, P. The Fen Damkjernite: Petrology of a "central-complex kimberlite". Phys. Chem. Earth 1975, 9, 163-177. [CrossRef]

72. Meert, J.G.; Torsvik, T.H.; Eide, E.A.; Dahlgren, S. Tectonic Significance of the Fen Province, S. Norway: Constraints from Geochronology and Paleomagnetism. J. Geol. 1998, 106, 553-564. [CrossRef]

73. Roberts, R.J.; Corfu, F.; Torsvik, T.H.; Ashwal, L.D.; Ramsay, D.M. Short-lived mafic magmatism at 560-570 $\mathrm{Ma}$ in the northern Norwegian Caledonides: U-Pb zircon ages from the Seiland Igneous Province. Geol. Mag. 2006, 143, 887-903. [CrossRef]

74. Brueckner, H.K.; Rex, D. K-A and Rb-Sr geochronology and Sr isotopic study of the Alnö alkaline complex, northeastern Sweden. Lithos 1980, 13, 111-119. [CrossRef]

75. Kresten, P. The Alnö complex: Tectonics of dyke emplacement. Lithos 1980, 13, 153-158. [CrossRef]

76. Robert, B.; Domeier, M.; Jakob, J. Iapetan Oceans: An analog of Tethys? Geology 2020, 48, 929-933. [CrossRef]

Publisher's Note: MDPI stays neutral with regard to jurisdictional claims in published maps and institutional affiliations. 